\title{
The profile structure for Luce's choice axiom
}

\author{
Donald G. Saari \\ Department of Economics, University of California, 3151 Social Science Plaza, Irvine CA 92697-5100, USA
}

Received 30 May 2003; received in revised form 17 July 2004

\begin{abstract}
A geometric approach is introduced to explain phenomena that can arise with Luce's choice axiom; e.g., differences occur when determining the likelihood of a ranking by starting with the "best-first," or "worst-first" alternative. As shown, the problem is caused by the way we compute pairwise probabilities: it forces "best-first" and "worst-first" computations to use different information from a profile. Thus agreement holds only should the different information agree: this happens only with complete indifference. An alternative "best-first" and "worst-first" comparison, which always holds, is developed. Ways to increase the applicability of the choice axiom are introduced; e.g., profiles admitted by Luce's formulation for ten alternatives have nine degrees of freedom; the approach described here allows millions of degrees of freedom. New ways to compute probabilities, which combine "best-first" and "worst-first" computations, are given: their properties are identified with a profile decomposition. A new way to compute pairwise probabilities, which eliminates all profile restrictions and problems associated with the choice axiom, is introduced; e.g., "best-first" and "worst-first" computations now agree. Three and four alternatives are emphasized for reasons of exposition, but most results extend to any number of alternatives.
\end{abstract}

(C) 2005 Elsevier Inc. All rights reserved.

\section{Introduction}

With the introduction of different axiomatic systems, one by Arrow (1963) for group processes and another by Luce $(1959,1961,1962,1977)$ for individual decisions, the 1950 s proved to be a seminal period for decision and choice theory. These approaches differ in fundamental ways: Arrow emphasized the properties of decision rules while Luce imposed a relationship on the outcomes. Luce then concentrated on a particular decision rule and its associated profiles. I adopt a different perspective: by using Luce's relationships to implicitly define all admissible decision rules and all of their associated profiles, my emphasis is on the structure of the space of profiles. In this manner, a far richer class of probability measures and profiles emerge that are compatible with Luce's choice axiom. Thus, the approach described here significantly increases the potential applicability of Luce's conditions.

E-mail address: dsaari@uci.edu.
To motivate both Arrow's and Luce's systems, suppose 78 voters rank the four alternatives $A, B, C, D$ as follows where " $\succ$ " means "is strictly preferred to."

\begin{tabular}{|c|c|c|c|}
\hline Number & Ranking & Number & Ranking \\
\hline 5 & $A \succ B \succ C \succ D$ & 9 & $B \succ D \succ A \succ C$ \\
\hline 7 & $A \succ C \succ B \succ D$ & 8 & $C \succ B \succ A \succ D$ \\
\hline 9 & $A \succ D \succ B \succ C$ & 11 & $C \succ D \succ A \succ B$ \\
\hline 4 & $B \succ A \succ C \succ D$ & 8 & $D \succ B \succ A \succ C$ \\
\hline 7 & $B \succ C \succ A \succ D$ & 10 & $D \succ C \succ A \succ B$ \\
\hline
\end{tabular}

The plurality election outcome (where each voter votes for one alternative) is $A \succ B \succ C \succ D$ with the $21: 20$ : $19: 18$ tally. Dropping any alternative flips the election outcome so that it now is compatible with the reversed $D \succ C \succ B \succ A$. (For instance, by dropping $D$, the new $C \succ B \succ A$ ranking has a $29: 28: 21$ tally.) But dropping any two alternatives flips the pairwise majority vote rankings to agree with the original $A \succ B \succ C \succ D$ outcome. 
To convert Eq. (1.1) into a individual decision problem, normalize (that is, divide each number by 78) to create a table that describes the probability a subject assigns to each ranking. This simple connection between aspects of voting theory and individual decisions allows us to use recent results from voting theory (Section 2) to uncover new conclusions about individual decision making. Creating such connections is a theme of this paper. For the rest of this introductory section, issues are raised, notation is introduced, and an outline for the paper is given.

\subsection{Some issues}

Although Eq. (1.1) example had nothing to do with Arrow's and Luce's initial motivation, it is useful to view their contributions as searching for ways to ensure consistent outcomes. But to achieve "consistency," it may be necessary to restrict preferences: an unfortunate consequence of restrictions is that they limit the applicability of a theory. A natural goal, then, is to find ways to relax restrictions so that a theory can admit other interesting settings. I identify alternative approaches that significantly relax the current restrictions; indeed, one approach imposes no restrictions.

To be more specific about Arrow's approach, his "binary independence" condition (or "IIA") can be thought of as constructing a societal ranking by using only the information about how each voter ranks each pair of alternatives. As Arrow proved, the only way to assure transitive societal rankings with his conditions is for the decision rule to be a dictatorship. The reason, as we now know (Saari, 1998, 2001b), is that if a decision rule satisfies binary independence, then it dismisses the explicitly specified information that the voters have transitive preferences. As described later, this "loss of transitivity" phenomenon occurs only with specific configurations of preferences. Interestingly, the data component that causes Arrow's problems is crucial for Luce's results (Section 6).

If binary independence dismisses the crucial assumption of transitive preferences, then a natural way to sidestep the difficulties Arrow identified is to relax his binary independence condition so that a decision rule can use the information that voters' preferences are transitive. By taking this approach (Saari, 1998, 2001b), Arrow's dictator is replaced with several rules including the Borda Count (BC): a decision rule that plays an interesting role in voting and in our discussion of Luce's axiom (Sections 3-7).

Definition 1. For $n$-alternatives, the $B C$ tallies each voter's ballot by assigning $(n-j)$ points to the voter's $j$ th ranked alternative, $j=1, \ldots, n$. The alternatives are ranked according to the sum of received votes.

\subsection{Luce's approach}

With his interest in individual decision making, Luce considers settings that can be captured with probability measures. For notation, letters in the early part of the alphabet such as $A, \ldots, D$, correspond to alternatives, $X, Y, Z$ are variables, $R, S, T$ represent sets, and lower case letters represent probabilities; e.g., $P(A)=a$. The probability measure $P_{T}$ satisfies the usual axioms for space $T$; i.e.,

(1) For $S \subset T, 0 \leqslant P_{T}(S) \leqslant 1$.

(2) $P_{T}(\emptyset)=0, P_{T}(T)=1$.

(3) If $R, S \subset T$ and $R \cap S=\emptyset$, then $P_{T}(R \cup S)=$ $P_{T}(R)+P_{T}(S)$.

For $S \subset T, P_{S}$ denotes the conditional probability measure defined over set $S$ and $P(A, B)$ represents $P_{\{A, B\}}(A)$ : the probability of selecting $A$ in $S=\{A, B\}$.

Luce avoids Eq. (1.1) type of inconsistencies by requiring consistent outcomes where the probabilities in any set $T$ determine all consistent subset outcomes. He accomplishes this feat by imposing the following conditions that are called the "choice axiom" in what follows.

Axiom 1. Luce's Choice Axiom (Luce, 1959). For any $n \geqslant 2$, let $T=\left\{A_{1}, A_{2}, \ldots, A_{n}\right\}$ be a set of $n$ alternatives. $A$ probability measure $P_{T}$ satisfies the choice axiom if the following are true.

(1) For every non-empty $S \subset T, P_{S}$ is defined.

(2) If $P\left(A_{i}, A_{j}\right) \neq 0,1$ for all $A_{i}, A_{j} \in T$, then for $R \subset S \subset T$

$$
P_{T}(R)=P_{S}(R) P_{T}(S) \text {. }
$$

(3) If $P\left(A_{i}, A_{j}\right)=0$ for some $A_{i}, A_{j} \in T$, then for every $S \subset T$

$$
P_{T}(S)=P_{T-\left\{A_{i}\right\}}\left(S-\left\{A_{i}\right\}\right) .
$$

Because $P(A, B)=0$ implies that $P(B, A)=1$, (3) describes an individual with perfect discrimination between alternatives $A_{i}$ and $A_{j}$. Luce $(1959$, p. 8) imposed this condition to avoid questionable behavior that can be associated with perfect discrimination. While I also emphasize imperfect discrimination (so, for all $A, B \in T$, assume that $P(A, B) \neq 0,1)$, I suggest, in Section 7, why Condition (3) is necessary.

With imperfect discrimination, conditional probability is defined as

$P_{T}(R \mid S)=\frac{P_{T}(R \cap S)}{P_{T}(S)}$.

Luce noted for $R \subset S \subset T$ that $P_{S}(R)=P_{T}(R \mid S)$ is equivalent to Eq. (1.2), so his axiom is equivalent to the 
standard independence relationship. A subtle but important distinction is that Luce does not require an universal set, so his setting holds for those realistic situations where the set of alternatives changes. Namely, alternatives can be added to the system without needing to renormalize to ensure that the sum of the probabilities equals unity. A central reason why this property holds is that, as described below, Luce's axiom has the effect of endowing each alternative with an intrinsic level of likelihood that is independent of the particular set. Thus, while $P_{T}$ denotes a probability defined over set $T$, this notation does not imply that $T$ is the universal set.

\subsection{Rankings}

I consider only settings where a subject ranks alternatives. Following Luce (1959, pp. 68-74), for the set of alternatives $T$, let " $P_{T}(A)$ denote the probability that $A$ is judged to be the superior element in $T$ according to some specified criterion." The reason for the "some specified criterion" phrase is that, as Luce states and as I show (Sections 3 and 7), selecting a criterion is equivalent to adding conditions to the choice axiom. But independent of what criterion is selected, the choice axiom and standard probability conditions force the following structure. (Luce uses a more general representation.)

Proposition 1 (Luce, 1959). Assume the choice axioms hold. For $S \subset T$ and $A_{i} \in S$,

$P_{S}\left(A_{i}\right)=\frac{P_{T}\left(A_{i}\right)}{\sum_{A_{j} \in S} P_{T}\left(A_{j}\right)}$.

As Proposition 1 demonstrates, Luce's conditions impose an orderly structure on the outcomes where the $\left\{P_{T}\left(A_{j}\right)\right\}_{j=1}^{n}$ values determine all remaining $P_{S}\left(A_{j}\right)$ probabilities. Notice how Eqs. (1.4) and (1.5) provide the intended sense that attached to each alternative $A_{j}$ is an intrinsic $P_{T}\left(A_{j}\right)$ level of likelihood. This property, for instance, means that $P_{T}$ can be constructed from the pairwise probabilities: this is illustrated next for $T=\{A, B, C\}$.

Proposition 2 (Luce, 1959, p. 16). If $X, Y, Z$ represent the three alternatives of $T=\{A, B, C\}$, then, under the assumption of imperfect discrimination,

$P_{T}(X)=\frac{P(X, Y) P(X, Z)}{P(X, Y)+P(X, Z)-P(X, Y) P(X, Z)}$

and

$P(B, C)=\frac{P(A, C) P(B, A)}{P(A, B) P(C, A)+P(B, A) P(A, C)}$.

So, the probability of selecting $X$ from $T$ is determined by how $X$ fares in both pairwise comparisons. A related relationship holds for $n \geqslant 4$.
Proof. Let $P_{T}(A)=a, P_{T}(B)=b, P_{T}(C)=c$. Substitute the $P(X, Y)=\frac{x}{x+y}$ values into these expressions and collect terms.

While the choice axiom specifies relationships among outcomes, observe that it does not even mention the decision rules. This suggests exploring whether the choice axiom can be treated as implicitly defining a class of admissible decision rules (i.e., probability computations) and their associated profiles. Carrying out this program is a main theme of the paper. First a "profile" must be defined.

Definition 2. For $n \geqslant 3$ alternatives, there are $n$ ! strict (complete, linear) transitive rankings. A voting profile lists the number of voters who have each ranking as a personal preference. A probability profile $\mathbf{p}$ lists the probability that a particular ranking occurs. That is, each component of $\mathbf{p}$ is non-negative and the sum of the components is unity.

With a voting profile, ballots are tallied. With a probability profile, the various ranking probabilities are determined by the summation rules of probability. To illustrate, when Eq. (1.1) is converted into a probability profile (by dividing each number by the total number of votes), the inconsistency of Eq. (1.1) rankings over different subsets means that if the probability measure is equivalent to the plurality vote, then profiles of Eq. (1.1) type are not admitted by the choice axiom. (This statement is an immediate consequence of the fact that a profile satisfying Luce's axiom assigns an "intensity of likelihood" value to each alternative. This fixed intensity requires the alternatives to retain the same relative ranking with different subsets; e.g., profiles that satisfy Luce's axiom also satisfy Arrow's binary independence condition.) If Eq. (1.1) profile is not allowed, what is? The admitted profiles are characterized starting in Section 3.

The choice axiom introduces intriguing mysteries when constructing $T=\{A, B, C\}$ rankings. One of them compares the effects of an individual constructing a ranking with a "best-first" approach versus a "worstfirst" method. For a "best-first" approach, it is reasonable for the likelihood of $A \succ B \succ C$, denoted by $R(A \succ$ $B \succ C$ ), to be

$R(A \succ B \succ C)=P_{T}(A) P(B, C)$.

Namely, the subject first selects the top ranked $A$ with likelihood $P_{T}(A)$, and then selects $B$ from the remaining two alternatives with probability $P(B, C)$. In contrast, a worst-first approach starts with likelihood that $C$ is judged to be inferior, $P_{T}^{*}(C)$, and then selects $B$ as the inferior choice of the remaining two with likelihood 
$P^{*}(B, A)$ to obtain

$R^{*}\left(A \succ^{*} B \succ^{*} C\right)=P_{T}^{*}(C) P^{*}(B, A)$,

where $P_{T}^{*}$ satisfies the choice axiom with $P^{*}(X, Y)$.

Under Luce's reasonable assumption that pairwise decisions for either approach agree, that is, $P(A, B)=$ $P^{*}(B, A)$, one might expect $\quad R(A \succ B \succ C)=$ $R^{*}\left(A \succ^{*} B \succ^{*} C\right)$. But Luce proves that this relationship requires a peculiar restrictive condition.

Theorem 1 (Luce, 1959, p. 69). Let $P$ and $P^{*}$ be defined as above for $T=\{A, B, C\}$, and assume that they both satisfy the choice axiom where all pairwise discriminations are imperfect and $P(X, Y)=P^{*}(Y, X)$. A necessary and sufficient condition for $R(A \succ B \succ C)=R^{*}\left(A \succ^{*} B \succ^{*} C\right)$ is that $P(A, B)=P(B, C)$.

This unexpected behavior is nicely examined by Yellott(1997a,b) in terms of Thurstone's theory of comparative judgment. Other insightful papers in this direction include Fishburn (1994), Marley (1982), and papers in the edited book (Marley, 1997). Estes (1997) describes the flavor of the thinking of the time when Luce's theorem and choice axiom were discovered. The discussion of Theorem 1 given here differs and explains this condition in several ways.

\subsection{Debreu, Tversky, and reversal problems}

While Theorem 1 is discussed throughout this paper, it admits a particularly simple "number of equations and number of unknowns" explanation. A way to motivate the argument is to recall Debreu's (1960) criticism of the choice axiom in a review of Luce's book and to show why Tversky's elimination-by-aspect approach is subject to the same difficulty.

To capture the flavor of Debreu's example with alternatives $T=\{A, B, C\}$, let $A$ and $B$ represent the same CD featuring Sibelius, but from different stores, so I am indifferent between them. Let alternative $C$ be a CD featuring Mozart: I am indifferent between purchasing a CD featuring Mozart or Sibelius. Thus, the likelihood of selecting either alternative from any pair is a half. But according to Eq. (1.6), the choice axiom requires the unreasonable $P_{T}(A)=P_{T}(B)=P_{T}(C)=\frac{1}{3}$. A more realistic choice has an equal likelihood for selecting either a Mozart or Sibelius CD where the Sibelius choices split to define $P_{T}(A)=P_{T}(B)=\frac{1}{4}$ while $P_{T}(C)=\frac{1}{2}$. Debreu argues that, "To meet this difficulty one might say that the alternatives have not been properly defined. But how far can one go in the direction of redefining the alternatives to suit the axiom without transforming the latter into a useless tautology?"

Debreu's comment are recalled only to introduce the "equation counting" argument used throughout this paper. The main point is that the choice axiom is intended to model only those settings where a natural intensity is attached to each alternative: in contrast, Debreu's example captures a natural multi-dimensional situation where the intensity changes with the setting. Restated in terms of the number of equations and number of unknowns, by attaching an intensity to each alternative, Luce's axiom allows $(n-1)$ degrees of freedom for $n \geqslant 3$ alternatives. Consequently, the choice axiom can be used only for settings that can be described with no more than $n-1$ independent expressions (equations). (The $n$ choices of $P_{T}\left(A_{j}\right), j=1, \ldots, n$, are constrained by $\sum_{j} P_{T}\left(A_{j}\right)=1$.) Debreu's example involves five independent expressions, so, rather than a criticism of the choice axiom, his example underscores the reality that we cannot model situations with more independent expressions than allowed degrees of freedom.

It is interesting, and may have been noticed by others, that a similar restriction applies to Tversky's elimination-by-aspect method (Tversky, 1972a,b). This is because with $n$ alternatives the number of aspects defines $2^{n}-2$ independent variables coming from the $2^{n}$ possible subsets minus the empty set and the set where all aspects agree. Each of the $\left(\begin{array}{l}n \\ j\end{array}\right)$ subsets with $n-j$ alternatives define $(n-j-1)$ different $P_{S}\left(A_{j}\right)$ probabilities. Thus, over all subsets of alternatives, it is possible to describe up to

$\tau(n)=\sum_{j=0}^{n-2}\left(\begin{array}{l}n \\ j\end{array}\right)(n-j-1)$

different independent expressions. Consequently, once $\tau(n)>2^{n}-2$, which occurs already for $n \geqslant 4$, examples with the Debreu flavor can be constructed where Tversky's elimination-by-aspect framework is not applicable. An example requires more than $2^{n}-2$ expressions, and Eq. (1.1) example indicates how to construct such situations.

A more subtle twist on this "number of equations and number of unknowns" argument explains Theorem 1. To see this argument for $T=\{A, B, C\}$, let $x=$ $P_{T}(A), y=P_{T}(B)$, and $1-x-y=P_{T}(C)$. Similarly, let $u=P_{T}^{*}(A), v=P_{T}^{*}(B)$, and $1-u-v=P_{T}^{*}(C)$. With the choice axiom, the three conditions $P(A, B)=P^{*}(B, A)$, which is $\frac{x}{x+y}=\frac{v}{u+v}, P(A, C)=P^{*}(C, A)$, and $P(B, C)=$ $P^{*}(C, B)$ create two constraints for the four variables. This leaves two degrees of freedom. (There are two independent constraints because, according to Eq. (1.7), the probabilities for any two pairs uniquely determines those for the third.) Now consider the central relationship

$$
\begin{aligned}
& R(A, B, C)-R^{*}(A, B, C) \\
& \quad=x\left(\frac{y}{y+(1-x-y)}\right)-(1-u-v)\left(\frac{v}{u+v}\right)
\end{aligned}
$$


that we hope equals zero. But Eq. (1.11) is a single equation with two degrees of freedom, so instead of just the zero value (indicating equality) we must expect a continuum of values. In particular, Eq. (1.11) has the desired unique zero value only after imposing another independent condition (equation) to have the correct number of equations and unknowns. The seemingly strange $P(A, B)=P(B, C)$ requirement of Theorem 1 is one choice for this extra condition; there are others (Section 5).

The real objective is to have all $R(X, Y, Z)=$ $R^{*}(X, Y, Z)$ probabilities agree. By repeatedly using Theorem 1 with all six rankings, all six $R(X, Y, Z)=$ $R^{*}(X, Y, Z)$ probabilities agree if and only if

$P(A, B)=P(B, C)=P(C, A)$,

$P(B, A)=P(A, C)=P(C, B)$,

where the terms for the first string of equalities come from achieving agreement with the rankings

$A \succ B \succ C, \quad B \succ C \succ A, \quad C \succ A \succ B$

and those for the last string come from $B \succ A \succ C, A \succ$ $C \succ B, C \succ B \succ A$.

Eq. (1.13) configurations are called Condorcet triplets, and, as described starting in Section 2, they play a central role in voting theory, probability computations, Arrow's theorem, and the choice axiom. According to Eq. (1.12), if $R(\sigma)=R^{*}(\sigma)$ for all rankings $\sigma$, then there is at most one degree of freedom, say the $\frac{a}{a+b}$ value. An equation counting argument shows that the actual restriction is more severe; all rankings have probability $\frac{1}{6}$. This is what Luce (1959) (also see Luce, Buch, \& Galanter, 1965) proved; different explanations using information about profiles are given in Sections 5 and 6.

\subsection{Pairwise computations}

There is no fixed definition for the "likelihood of a pairwise ranking," but the intuitive approach defines $P(A, B)$ as the sum of probabilities over rankings where $A \succ B$. This approach is not necessary: probability computations such as the one given later in Definition 10 could assign more weight to rankings such as $A \succ C \succ B$, which separate $A$ and $B$, than to $A \succ B \succ$ $C$ where $A$ and $B$ are adjacent.

For notation, if $S \subset T$ and $\sigma$ is a ranking of the alternatives in $S$, let $R_{S}(\sigma)$ denote the probability of $\sigma$. It follows from Eq. (1.6) that a probability $P_{T}^{\prime}(X)$ (see Eq. (7.9)) can be constructed that satisfies the choice axiom where

$$
\begin{aligned}
P^{\prime}(A, B) & =\sum_{\rho \text { where } A \succ B} \frac{5}{6} R_{T}(\rho)+\sum_{\rho \text { where } B \succ A} \frac{1}{6} R_{T}(\rho) \\
& =\frac{1}{6}+\left[\frac{2}{3} \sum_{\rho \text { where } A \succ B} R_{T}(\rho)\right] .
\end{aligned}
$$

Observe that $P^{\prime}$ and the standard approach always agree in how the alternatives are ranked according to likelihoods, but the values differ; e.g., $P^{\prime}$ never allows perfect discrimination. A natural question is to determine when we must use the standard approach to compute $P(A, B)$.

Theorem 2 (Luce (1959)). Let $R_{S}$ and $P_{S}$ be defined for all $S \subset T$ and suppose that

(1) $R_{\{A, B\}}(A \succ B)=P(A, B), R_{T}(A \succ \rho)=P_{T}(A) R_{T-\{A\}}(\rho)$,

(2) $P_{S}$ satisfies the choice axiom,

(3) all pairwise discriminations are imperfect;

then

$P(A, B)=\sum_{\rho \text { where } A \succ B} R_{T}(\rho)$.

Call Eq. (1.15) the "standard pairwise computation." The next more general definition is used in what follows.

Definition 3. Let $\sigma$ be a ranking of the alternatives in $S \subset T$. The linear computation of $R_{S}(\sigma)$ is where $R_{S}(\sigma)$ is a specified linear combination of $R_{T}(\rho)$ for all rankings $\rho$. The standard computation of $R_{S}(\sigma)$ is the special case where

$R_{S}(\sigma)=\sum_{\rho \text { where } \sigma \text { is the relative ranking }} R_{T}(\rho)$.

Eq. (1.14) illustrates a particular choice for a nonstandard linear pairwise computation: there exist many other reasonable ways, even nonlinear approaches, to compute these probabilities. While it is interesting to speculate whether individuals use other computational approaches, the standard pairwise approach is emphasized until Definition 10: as we will see, this Definition 10 approach to pairwise comparisons eliminates most of the problems that are associated with the choice axiom.

\subsection{The "Ranking Axiom"}

Comparing $P(A, B)$ with $P^{\prime}(A, B)$ from Eq. (1.14) shows that there exist rules over different subsets of alternatives where the numerical scores for alternatives may differ, but they always share the same ordinal ranking (based on the probabilities). Thus it is possible to relax the precision of the choice axiom while preserving its spirit: an advantage of doing so is to admit settings where a subject does not exhibit Luce's required numerical precision. This alternative axiom, which captures the spirit of Arrow's and Luce's approaches, imposes consistency about how a subject ranks alternatives without requiring precise probability values.

Axiom 2 (Ranking Axiom). For the alternatives $T=$ $\left\{A_{1}, \ldots, A_{n}\right\}$, let $S$ denote a subset. Suppose $P$ is a decision 
rule defined over all subsets of alternatives where $P_{S}$ represents the rule restricted to subset $S$. Decision rule $P$ and an associated subset of profiles $\mathscr{P} \mathscr{R}_{P}$ satisfies the ranking axiom if the following hold for each $S \subset T$, $|S| \geqslant 2$.

(1) For each $S$ and $\mathbf{p} \in \mathscr{P} \mathscr{R}_{P}, P_{S}(\mathbf{p})$ is a complete, binary, transitive ranking of the alternatives in $S$.

(2) The ranking of $P_{S}(\mathbf{p})$ is the same as the relative ranking of the alternatives from $S$ in $P_{T}(\mathbf{p})$.

If a probability measure satisfies the choice axiom for the alternatives in $T$, it also satisfies the Ranking Axiom. But as Eq. (1.14) demonstrates, a decision rule can fail the choice axiom and still satisfy the Ranking Axiom. (Also see standard results from random utility models.) Similarly, if a profile restriction admits a method where Arrow's Binary Independence holds for the ranking of all sets $S$, then it satisfies the Ranking Axiom. The added flexibility offered by this axiom is indicated in Section 6 where I introduce a continuum of settings with decision rules that satisfy this condition but not the choice axiom.

\subsection{Outline}

To outline this paper, in Section 2, I describe a recently developed approach to analyze voting rules. A way to think of this approach is that Arrow stresses the structure of the decision rules, Luce stresses the structure of the outcomes, while I stress the structure of the domain - the space of profiles. More specifically, this approach decomposes a voting or probability profile into those components that cause different outcomes when used by different kinds of rules. This decomposition helps to explain some of the mysteries that are associated with Luce's axiom.

In Sections 3 and 4, results from voting theory are used to create a class of new probability measures that satisfy the choice axiom and to prove that a surprisingly large set of probability profiles satisfy the choice axiom. By using these new probability measures, we find new ways to describe the concerns introduced in Theorem 1. As an illustration, a measure based on Definition 10 has several remarkable properties; e.g., no restrictions on profiles are needed to satisfy the choice axiom and many of the choice axiom difficulties disappear. In Section 5, different ways to model Theorem 1 reversal behavior are investigated: as true with Luce's approach, in all cases the ranking probabilities agree only at complete indifference. Section 6 uses the voting material introduced in Section 2 to explain the source of several of the choice axiom mysteries. In this section, I explain why the Condorcet triplet (Eq. (1.13)) that causes Arrow's negative conclusions plays a vital positive role for Luce's choice axiom.
In Section 7, I show that there remains an uncountable number of other probability measures that satisfy the choice axiom, and I identify other consequences of the choice axiom.

\section{A decomposition of profiles}

The purpose of this section is to describe recent results from voting theory that can be used to explain the source of all possible election outcomes that can arise with a large class of voting rules. When applied to probability measures and probability profiles, this theory explains a variety of the choice axiom behaviors including the earlier described reversal results. I start by showing how to list profiles in a geometric manner: the advantage of doing so is that the geometry sorts the profile entries in a manner to simplify the computations of election outcomes. To introduce the notions with $n=3$ alternatives, label the 3 ! strict, complete, transitive rankings as specified next.

$\begin{array}{cccc}\text { Type number } & \text { Ranking } & \text { Type number } & \text { Ranking } \\ 1 & A \succ B \succ C & 4 & C \succ B \succ A \\ 2 & A \succ C \succ B & 5 & B \succ C \succ A \\ 3 & C \succ A \succ B & 6 & B \succ A \succ C\end{array}$

The rankings of $n \geqslant 3$ alternatives can be geometrically represented with an equilateral $n$-gon that resides in a $(n-1)$-dimensional space. For $n=3$, this figure is an equilateral triangle; for $n=4$ it is an equilateral tetrahedron. Assign each alternative to an unique vertex, and assign rankings to each point in the equilateral $n$-gon according to the point's distance from each vertex where "closer is better." In Fig. 1a, for instance, any point in the small triangle with a " 1 " is closer to the $A$ vertex than the $B$ vertex, so it has a $A \succ B$ binary ranking. Points on Fig. 1a vertical line (connecting the midpoint of the $A-B$ edge with the $C$ vertex) are equidistance from $A$ and $B$, so they represent where $A$ is tied with (or indifferent to) $B$ denoted by $A \sim B$.

In this manner, the $n$-gon is divided into ranking regions. The numbers placed in the small triangles of Fig. 1a identify which ranking region has which Eq. (2.1) strict ranking; e.g., for a point in the small triangle with a "2," the closest vertex is $A$, the next closest vertex is $C$, and the farthest is $B$, so the assigned ranking is $A \succ C \succ B$. The remaining rankings include the complete indifference $A \sim B \sim C$ outcome (the triangle's barycenter) and six rankings with one pairwise indifference. These last six rankings are represented by the line segments that separate open triangular regions. (For instance, the line segment representing $A \succ B \sim C$ separates regions 1 and 2.) Indifference rankings are excluded to allow a cleaner exposition. 


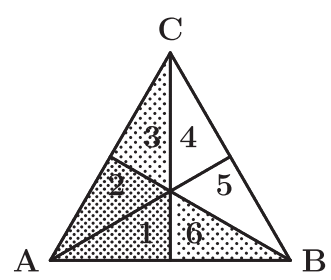

(a) Names for rankings

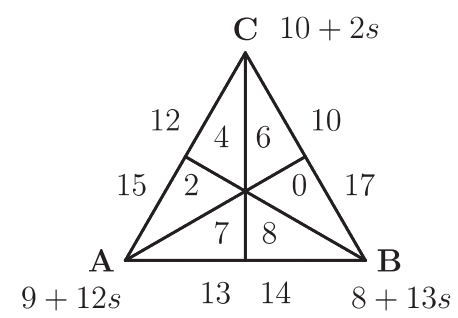

(b) Voting profile

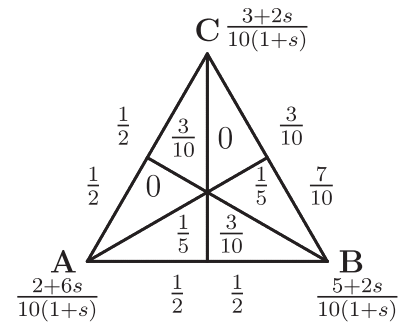

(c) Probability profile

Fig. 1. Representation triangle: (a) names for rankings, (b) voting profile and (c) probability profile.

\subsection{Profiles and tallying ballots}

With this notation, the "voting profile space" is

$$
\begin{aligned}
\mathscr{V} \mathscr{P}^{3}= & \left\{\mathbf{n}=\left(n_{1}, \ldots, n_{6}\right) \in R^{6} \mid n_{j}\right. \\
& \text { is a non-negative integer }\},
\end{aligned}
$$

where $n_{j}$ is the number of voters with the $j$ th preference ranking. It is difficult to envision this six-dimensional $\mathscr{V} \mathscr{P}^{3}$, so I use Fig. 1 triangle to represent $\mathscr{V}_{P^{3}}$ profiles by placing $n_{j}$ in the open region representing the $j$ th ranking, $j=1, \ldots, 6$. In this way, Fig. $1 \mathrm{~b}$ represents the profile $(7,2,4,6,0,8)$. Similarly, a "probability profile space" is

$$
\begin{aligned}
\mathscr{P}^{3}= & \left\{\mathbf{p}=\left(p_{1}, p_{2}, p_{3}, p_{4}, p_{5}, p_{6}\right) \in R^{6} \mid \sum_{j=1}^{6} p_{j}=1,\right. \\
& \left.p_{j} \geqslant 0 \forall j\right\},
\end{aligned}
$$

where $p_{j}$ is the probability that the $j$ th ranking occurs; $j=1, \ldots, 6$. Fig. $1 \mathrm{c}$ has the probability profile $\left(\frac{1}{5}, 0, \frac{3}{10}, 0, \frac{1}{5}, \frac{3}{10}\right)$. (For $n$-alternatives, use the obvious definitions for $\mathscr{V} \mathscr{P}^{n}$ and $\mathscr{P}^{n}$.)

To see how this geometric profile representation facilitates computing election tallies, notice that all voters preferring $A \succ B$ are represented by the numbers to the left-hand side of Fig. 1b vertical line; thus, $A$ 's tally in an $\{A, B\}$ election is the $4+2+7=13$ sum of numbers on this side. Similarly, $B$ 's tally is the $6+0+$ $8=14$ sum of numbers that are on the right-hand side. All pairwise tallies are similarly computed and listed by the appropriate edge of the triangle. Likewise, using the standard way to compute probabilities, e.g., $P(B, A)=p_{4}+p_{5}+p_{6}$, the computations for all pairs of Fig. 1c probability profile are posted next to the appropriate edge.

"Positional elections" (or "positional election rules") is the name attached to election rules where ballots are tallied by assigning points to candidates according to how they are positioned (i.e., ranked) on the ballot. The widely used "plurality vote," where each voter votes for one candidate and the candidate with the largest number of votes wins, assigns one point for a top-ranked candidate and zero for all others. The antiplurality vote is where one point is given to all but the last-ranked candidate who is assigned zero points.

Definition 4. For any choice of a positive integer $n \geqslant 2$, a $n$-alternative positional voting decision rule is defined by voting vector $\mathbf{w}^{n}=\left(w_{1}, w_{2}, \ldots, w_{n}\right), \quad w_{1}=1, w_{n}=0$, $w_{j} \geqslant w_{j+1}$ for $j=1, \ldots, n-1$, where $w_{j}$ points are assigned to a voter's $j$ th ranked alternative, $j=$ $1, \ldots, n$. The alternatives are ranked according to the sum of assigned points. In "positional probabilistic voting," the number of points assigned to each alternative for a specified ranking is multiplied by the probability of the ranking; the alternatives are ranked according to the sums. (To define "choice probabilities," further normalize the weights so that $\sum w_{j}=1$.)

To illustrate with the $\mathrm{BC}$, the normalized form and the form used for positional probabilistic voting are, respectively,

$\frac{1}{n-1}(n-1, n-2, \ldots, 1,0)$ and

$\frac{2}{n(n-1)}(n-1, n-2, \ldots, 1,0)$.

Using different choices of $\mathbf{w}^{n}$ to tally the same ballots can result in radically different election outcomes. With 10 candidates, for instance, a profile can be created whereby millions of different election rankings result by varying the choice of $\mathbf{w}^{10}$; indeed, it can be that each alternative wins with some decision rules but is bottomranked with others (Saari, 1992, 2000b). (The same assertion holds for probability profiles.)

Because different election rules can create different election rankings, a natural goal is to characterize all possible election outcomes that can result from all $\mathbf{w}^{n}$, to explain why each occurs, and to describe all supporting profiles: this project has been completed for any number of alternatives (Saari, 1989, 1999, 2000a,b). The $n=3$ results needed for our purposes are described next. (For details, proofs, and references, see Saari (1999, 2001a).)

The normalized three-candidate voting vector can be expressed as $\mathbf{w}_{s}=(1, s, 0)$ where $s$ is a specified 
value satisfying $0 \leqslant s \leqslant 1$; e.g., for any $s, A$ 's positional $\mathbf{w}_{s}$ tally is

[number of voters with $A$ top-ranked] $+s$ [number of voters with $A$ second-ranked].

Using Fig. 1a to represent a profile, the values for the first and second brackets would be the sum of numbers, respectively, from the heavily and the lightly shaded regions; e.g., with Fig. $1 \mathrm{~b}$ profile, $A$ 's $\mathbf{w}_{s}$ tally is $[2+7]+s[4+8]$. All $\mathbf{w}_{s}$ tallies are similarly computed and listed by the relevant vertex. The similar tallies for Fig. 1c probabilistic profile are further normalized to become probabilities by dividing by $1+s$.

The $s$-coefficients for Fig. $1 \mathrm{~b}$ election outcomes differ among the candidates, so it is reasonable to wonder whether the election outcomes can change by using different election rules. Indeed, candidate $A$ is the Borda winner $\left(s=\frac{1}{2}\right)$ where the Borda election ranking is $A \succ B \succ C, B$ is the Condorcet winner (she beats each of the other two candidates in pairwise majority vote elections), and $C$ is the plurality winner $(s=0)$ with the $C \succ A \succ B$ plurality outcome. In other words, each candidate can be the "winner" by using an appropriate election rule. A long-standing question in the area of social choice has been to understand and explain all conflicts of this sort. The basic ideas behind the approach answering these questions (see Saari (1999, 2001a) for $n=3$ candidate and Saari(2000a,b) for $n \geqslant 3$ candidates) are described next.

\subsection{Basic idea}

There are certain configurations of voter preferences where it is arguable that the outcome should be a complete tie. Indeed, if a voting rule fails to have a tie in such settings, it is reasonable to wonder whether the rule's tallies introduce a bias that favors certain candidates. This leads to the conjecture, which turned out to be true, that all possible election differences among standard voting rules are caused by these kinds of biases.

Surprisingly, for three candidates, only two configurations of preferences are needed to explain all possible differences in voting rules. The first is a reversal configuration where two voters have directly opposite rankings such as $A \succ B \succ C$ and $C \succ B \succ A$. Here it is arguable that the outcome should be a tie: this is what happens with the majority vote for any pair of alternatives. The reason is that if a pair is ranked one way in the first ranking, it is ranked in the opposite manner in the second ranking. For positional methods, however, a tie need not occur. Instead the $A: B: C \mathbf{w}_{s^{-}}$ tally is $1: 2 s: 1$. Consequently, the outcome is tied iff $s=\frac{1}{2}$, or iff the Borda rule is used. If $s<\frac{1}{2}$, as true with the plurality vote, the reversal configurations favor (or introduce a bias in favor of) $A \sim C$ over $B$. Conversely, choices of $s>\frac{1}{2}$ (such as the antiplurality rule) favor $B$ over $A \sim C$. Because the reversal configuration affects only positional methods, we might anticipate, and it is true, that all possible differences among positional methods are caused by profile configurations of this type. Moreover, because these components have no effect on pairwise rankings, they can cause conflicting election rankings among pairs and non-Borda positional rules.

To describe the next configuration, which I call the Condorcet $n$-tuple, start with any ranking, say $A_{1} \succ$ $A_{2} \succ \cdots \succ A_{n}$. To create the next ranking, move the topranked alternative to the bottom, so we have $A_{2} \succ \cdots \succ A_{n} \succ A_{1}$ : continue until all alternatives have been top-ranked once. To illustrate by starting with $A \succ B \succ C$, the Condorcet triplet, which already has cropped up with the choice axiom in Eq. (1.13), is

$A \succ B \succ C, \quad B \succ C \succ A, \quad C \succ A \succ B$.

Each candidate in the Condorcet triplet (and $n$-tuple) is ranked in each position precisely once, so it is arguable that the outcome should be a complete tie. The completely tied behavior is realized by all positional methods with their $A \sim B \sim C$ outcome where each candidate receives $1+s$ points. The pairwise vote, however, generates the cycle $A \succ B, B \succ C, C \succ A$ where each vote has a $2: 1$ tally. The reason the pairwise vote fails to have a tie is that by concentrating on pairs it cannot recognize nor utilize the broader symmetry that mandates a tied outcome. The cyclic tallies caused by this configuration bias pairwise election outcomes.

As one must anticipate, Condorcet triplets cause pairwise outcomes and tallies to differ from positional outcomes. The important fact (Saari, 2000a) is that the Condorcet $n$-tuples are completely responsible for all difficulties encountered in pairwise comparisons. As I have shown elsewhere, for instance, the Condorcet $n$ tuples are totally responsible for Arrow's dictator conclusion; e.g., by restricting attention to profiles with no Condorcet $n$-tuple components, Arrow's dictator is replaced with the $\mathrm{BC}$.

A way to illustrate how these biases change election outcomes is to show how Fig. 1b profile was constructed. Start with the innocuous Fig. 2a profile where its various tallies are listed by the edges and vertices: with the exception of the antiplurality vote $(s=1)$, all pairwise and positional tallies agree with the $A \succ B \succ C$ outcome. To change the Condorcet winner from $A$ to $B$ without affecting any $\mathbf{w}_{s}$-ranking, we know from the above that we must add Condorcet triplets. So, as represented in Fig. 2b, introduce $x$ units of the Condorcet triple $\{B \succ A \succ C, A \succ C \succ B, C \succ B \succ A\}$ that favors $B$ over $A$. By adding the pairwise tallies of Figs. $2 \mathrm{a}$ and $\mathrm{b}$, the combined profile has the pairwise majority vote rankings $B \succ A$ and $B \succ C$ iff the 

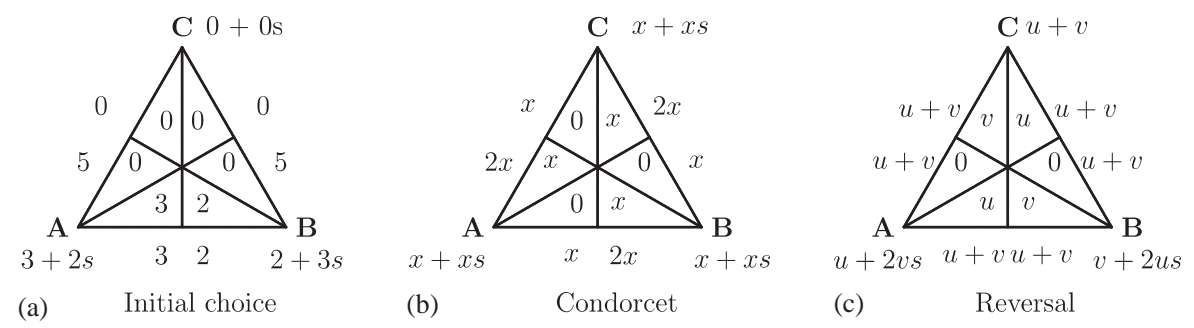

Fig. 2. Creating an example: (a) initial choice, (b) Condorcet and (c) reversal.

inequalities $2+2 x>3+x, 5+x>2 x$ are satisfied; e.g., iff $1<x<5$. I used $x=2$.

To change the positional outcomes without affecting pairwise and Borda outcomes, we now know that we must add reversal configurations. Select a ranking for any positional method: I selected $C \succ A \succ B$ for the plurality vote $(s=0)$. To favor $C$, use the two reversal configurations (Fig. 2c) where $C$ is top-ranked. Adding the values by the vertices of Figs. 2a and c (with $s=0$ ), this plurality election outcome occurs iff $u+v+0>3+$ $u>2+v$, or $v>3, u>v-1$. To create Fig. $1 \mathrm{~b}$ profile, use $u=v=4$.

In this simple manner, profiles exhibiting any collection of admissible election outcomes can be created. Moreover, all differences in three-alternative election outcomes can be explained solely in terms of the reversal and Condorcet components. The important point is that the reversal configurations affect only the positional outcomes, while the Condorcet configurations affect only the pairwise outcomes. This interaction between the two types of configurations of preferences will arise in much of what follows, including the choice axiom.

\subsection{Profile decomposition}

To convert the above description into an analytical tool, a coordinate system on the space of profiles is introduced. To keep the description simple, I consider only three candidates and jump between a profile with integer components (from $\mathscr{V} \mathscr{P}^{3}$ ) and its probabilistic representation (from $\mathscr{P}^{3}$ ). The approach just creates coordinates that reflect the reversal, the Condorcet triplets, and what remains. The origin of the coordinate system for $\mathscr{P}^{3}$ is at the neutral point denoted by $\mathbf{p}_{\mathrm{Neu}}=$ $\left(\frac{1}{6}, \ldots, \frac{1}{6}\right)$.

If $\mathbf{p}_{1}, \mathbf{p}_{2} \in \mathscr{P}^{3}$, then the sum of the components of the difference $\mathbf{p}_{1}-\mathbf{p}_{2}$ is zero. So vector $\mathbf{p}_{1}-\mathbf{p}_{2}$ lies in the tangent plane for $\mathscr{P}^{3}$ denoted by $\mathscr{T} \mathscr{P}^{3}$. (Treat $\mathscr{T} \mathscr{P}^{3}$ as the plane passing though the origin of $R^{6}$ that is orthogonal to $\mathbf{p}_{\mathrm{Neu}}$.) The following definition provides a name for the difference between any two probability profiles.

Definition 5. A profile differential is a vector in $R^{6}$ where the sum of its components equals zero.
For motivation, notice that the 24-voter profile $(3,2,7,1,5,6)$ can be represented as $(3,2,7,1,5,6)=$ $(4,4,4,4,4,4)+(-1,-2,3,-3,1,2)$; i.e., think of this sum as starting with a Neutral profile where the profile differential reassigns voters from some preference rankings (with negative signs) to others (with positive signs) to create the desired profile. The coordinate system for profile space is described with profile differentials.

Definition 6 (Saari, 1999). An $A_{j}$-basic profile differential, $\mathbf{B}_{A_{j}}$, assigns one point to each ranking where $A_{j}$ is top-ranked and -1 points to each ranking where $A_{j}$ is bottom-ranked. The basic profile space, consisting of all basic profile differentials, is the $\mathscr{T} \mathscr{P}^{3}$ subspace spanned by $\left\{\mathbf{B}_{A_{j}}\right\}_{j=1}^{3}$.

The $A_{j}$-reversal profile differential, $\mathbf{R}_{A_{j}}$, assigns one point to each ranking where $A_{j}$ is top or bottom-ranked and -2 points to each ranking where $A_{j}$ is middle ranked. The reversal subspace, consisting of all reversal profile differentials, is the $\mathscr{T} \mathscr{P}^{3}$ subspace spanned by $\left\{\mathbf{R}_{A_{j}}\right\}_{j=1}^{3}$.

The Condorcet profile differential, $\mathbf{C}$, assigns one point to each of the rankings in the Condorcet triplet $A \succ B \succ C, B \succ C \succ A, C \succ A \succ B$, and -1 points to each of the three remaining rankings that also form a Condorcet triplet.

The profile differentials for alternative $A$ are illustrated in Fig. 3. In Fig. 3, the pairwise tallies use the standard pairwise computation; the $\mathbf{w}_{s}$-outcomes, if not all zero, are listed next to the appropriate vertices. As it should be clear, the $\mathbf{R}_{X}$ reversal terms form a basis for all reversal configurations of the $A \succ B \succ C$ and $C \succ B \succ A$ type while the Condorcet term $\mathbf{C}$ is based on the Condorcet triplet. As described above, these differentials lead to a complete tie with certain voting rules, but they do not with other rules. These differences, which explain all of the positional-pairwise conflicts, is captured by Fig. 3 tallies.

Theorem 3 (Saari, 1999). Vectors from the basic, reversal, and Condorcet spaces are mutually orthogonal. The basic and reversal subspaces are two-dimensional; they are spanned by any two of the three defining vectors. Space $\mathscr{T}^{3}$ is spanned by the basic, reversal, and 


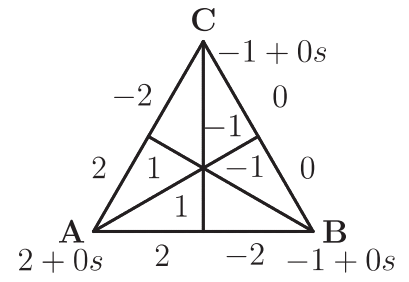

(a)

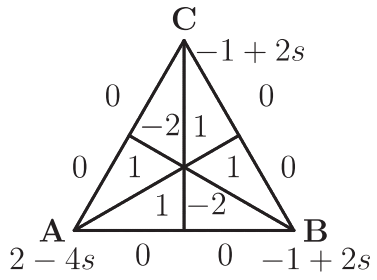

(b) A-Reversal

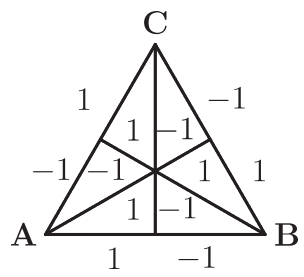

(c) Condorcet

Fig. 3. Profile decomposition: (a) $A$-Basic, (b) $A$-Reversal and (c) Condorcet.

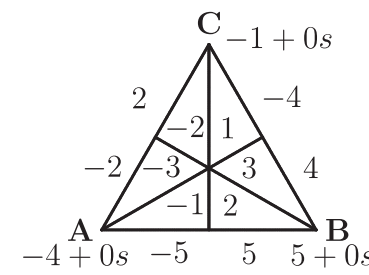

(a) $\quad \mathbf{p}_{B a}=3 \mathbf{B}_{B}+\mathbf{B}_{C}$

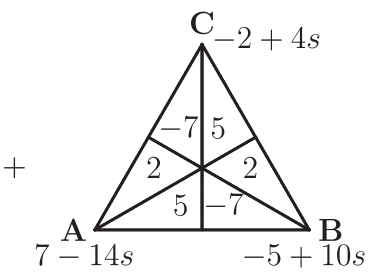

(b) $\quad \mathbf{p}_{\text {Rev }}=4 \mathbf{R}_{A}+\mathbf{R}_{C}$

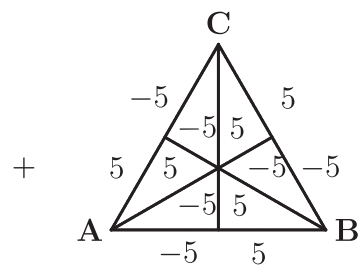

(c) $\mathbf{p}_{\text {Con }}=-5 \mathrm{C}$

Fig. 4. Constructing an example: (a) $\mathbf{p}_{\mathrm{Ba}}=3 \mathbf{B}_{B}+\mathbf{B}_{C}$, (b) $\mathbf{p}_{\mathrm{Rev}}=4 \mathbf{R}_{A}+\mathbf{R}_{C}$ and (c) $\mathbf{p}_{\mathrm{Con}}=-5 \mathbf{C}$.

Condorcet spaces. The properties of these differentials follow.

(1) All $\mathbf{w}_{s}$ tallies of a basic profile agree; for any $\mathbf{w}_{s}$ method, the common $\mathbf{B}_{A_{j}}$ tally assigns 2 points to $A_{j}$ and -1 points to each of the two remaining alternatives. The $\mathbf{w}_{s}$ ranking also is consistent with the rankings of the pairs when using the standard pairwise computation; this pairwise computation for $\mathbf{B}_{A_{j}}$ over a pair including $A_{j}$ assigns 2 points to $A_{j}$ and -2 points to the other alternative. If the pair does not involve $A_{j}$, both alternatives receive zero points.

(2) All pairwise tallies of a reversal differential end in 0:0 ties. Similarly, the Borda tally (using $\mathbf{w}_{1 / 2}$ ) is a complete tie where each alternative receives zero points. For all remaining $\mathbf{w}_{s}$, the outcome is determined by the relationships were the $\mathbf{w}_{s}$ tally for $\mathbf{R}_{A_{j}}$ assigns $2-4$ s points to $A_{j}$ and $2 s-1$ points toeach of the other two alternatives. Consequently, for s greater than, or less than, $\frac{1}{2}$, the ranking reverses.

(3) All $\mathbf{w}_{s}$ tallies of $\gamma \mathbf{C}$ are a complete tie where each alternative receives zero points. The pairwise computation of $\mathbf{C}$ creates a cycle whereby $A \succ B, B \succ$ $C, C \succ A$ all by $1:-1$ tallies.

According to Theorem 3, any three-alternative probability profile can be uniquely expressed as

$\mathbf{p}=\mathbf{p}_{\mathrm{Neu}}+\mathbf{p}_{\mathrm{Ba}}+\mathbf{p}_{\mathrm{Rev}}+\mathbf{p}_{\mathrm{Con}}$,

where the Ba, Rev, Con subscripts identify a profile component in, respectively, the Basic, Reversal, and Condorcet $\mathscr{T} \mathscr{P}^{3}$ subspaces. As Theorem 3 asserts, the $\mathbf{p}_{\mathrm{Ba}}$ differential has the delightful property that all positional and pairwise decision rules agree: no conflict can occur in tallies or rankings. Consequently, knowing the $\mathbf{w}_{0}$ plurality tallies for the $\mathbf{p}_{\mathrm{Ba}}$ term determines its $\mathbf{w}_{s}$ tallies for all $s$ (they all agree) and all pairwise outcomes. Conversely, the pairwise tallies can be used to determine all $\mathbf{w}_{s}$ tallies.

Since $\mathbf{p}_{\mathrm{Ba}}$ does not allow any conflict in outcomes among decision rules and subsets of alternatives, it follows that all conflict among decision rules and subsets of alternatives is caused by the components $\mathbf{p}_{\mathrm{Con}}$ (for the pairwise tallies) and $\mathbf{p}_{\mathrm{Rev}}$ (for the positional outcomes). The $\mathbf{p}_{\text {Con }}$ terms distorts the pairwise outcomes by adding a cyclic effect to the values. As Theorem 3 indicates and as shown earlier, the $\mathbf{p}_{\text {Rev }}$ term has opposing effects on outcomes depending on whether $s<\frac{1}{2}$, as with the plurality system, or $s>\frac{1}{2}$, as with the antiplurality approach.

A way to illustrate Theorem 3 is to create a probability profile with the plurality ranking of $A \succ B \succ C$, pairwise rankings that reflect the opposite $C \succ B \succ A$, and the Borda ranking $B \succ C \succ A$. Since only the Borda method is immune to the $\mathbf{p}_{\mathrm{Rev}}$ and $\mathbf{p}_{\mathrm{Con}}$ terms (Theorem 3), the desired Borda $B \succ C \succ A$ ranking requires using a Basic component such as $\mathbf{p}_{\mathrm{Ba}}=$ $3 \mathbf{B}_{B}+\mathbf{B}_{C}=(-1,-3,-2,1,3,2)$. (See Fig. 4a.)

According to Theorem 3, the only way the plurality ranking can differ from the Borda outcome is to add appropriate $\mathbf{p}_{\mathrm{Rev}}$ terms to the profile. In doing so, notice that the plurality tallies of $\mathbf{p}_{\mathrm{Ba}}+x \mathbf{R}_{C}+y \mathbf{R}_{A}$ for $A, B, C$ are, respectively, $-4-x+2 y, 5-x-y$, and $-1+2 x-y$. Thus a plurality $A \succ B \succ C$ ranking requires choosing $y>3$ and $2>x \geqslant 0$. The $x=1, y=4$ choices define the $\mathbf{p}_{\text {Rev }}$ illustrated in Fig. 4b. According to Theorem 3, $\mathbf{p}_{\text {Rev }}$ does not affect the Borda nor the pairwise rankings. 
The desired conflict in pairwise rankings can occur only by adding a Condorcet component. By computing the $\mathbf{p}_{\mathrm{Ba}}$ pairwise outcomes, and using Theorem 3 values for Condorcet outcomes, simple algebra shows that adding $\mathbf{p}_{\mathrm{Con}}=-5 \mathbf{C}$ to $\mathbf{p}_{\mathrm{Ba}}$ (Fig. $4 \mathrm{c}$ ) generates the desired pairwise rankings; $\mathbf{p}_{\text {Con }}$ does not affect the positional rankings.

As $\mathbf{p}_{\mathrm{Ba}}+\mathbf{p}_{\mathrm{Rev}}+\mathbf{p}_{\mathrm{Con}}=(-1,4,-14,11,0,0)$ (add the values for each region from Fig. 4 triangles), the components become nonnegative by adding 14 to each term: this corresponds to adding the Neutral profile. The total is $(13,18,0,25,14,14)$, so a probability profile with the desired properties is

$\mathbf{p}=\left(\frac{13}{84}, \frac{18}{84}, 0, \frac{25}{84}, \frac{14}{84}, \frac{14}{84}\right)$.

Recall from Theorem 3 that the $\mathbf{w}_{s}$ outcome for $\mathbf{p}_{\mathrm{Rev}}$ has one ranking for $s<\frac{1}{2}$ and the opposite ranking for $s>\frac{1}{2}$. Consequently, we should expect that similar phenomenon occurs for any profile with a sufficiently large $\mathbf{p}_{\text {Rev }}$ component. This property is satisfied by the above p: the plurality tally is $\left(\frac{31}{84}, \frac{28}{84}, \frac{25}{84}\right)$ with an $A \succ B \succ C$ ranking while the antiplurality tally is $\left(\frac{45}{168}, \frac{56}{168}, \frac{57}{168}\right)$ with the opposite $C \succ B \succ A$ ranking.

\section{Ranking probabilities}

The structure of voting procedures helps to analyze Luce's choice axiom with respect to ranking probabilities. (In what follows, most proofs for statements are in Section 9.) For other papers on ranking probabilities and related questions, see Fishburn (1994, 2002), Luce (1959, 1961, 1962, 1977), Marley (1968, 1982), and Yellott(1997a,b).

Luce claims that his "best-first" (Eq. (1.8)) model, where

$\mathscr{R}(A \succ B \succ C)=a \times \frac{b}{b+c}=a \frac{b}{1-a}$,

"is, in spirit, closely related to [the choice axiom]; however, it is logically independent of it." A quick way to recognize that there is a logical independence (for $n=3$ ) is to use a "number of equations and number of unknowns" argument. By doing so, it follows that the choice axiom does not introduce enough independent equations to determine the six ranking probabilities in a way to satisfy the choice axiom. Thus, a way to interpret the refined Eq. (3.1) probabilities is that they impose new assumptions, or, equivalently, they add new independent equations to the system. Denote Luce's profile by

$\mathbf{p}_{L}(a, b)=\left(\frac{a b}{1-a}, \frac{a c}{1-a}, \frac{c a}{1-c}, \frac{c b}{1-c}, \frac{b c}{1-b}, \frac{b a}{1-b}\right)$,

$c=1-a-b$.
By specifying Eq. (3.1) ranking probabilities, there is only one way to compute the associated probability values of $P_{T}(X)=x, X=A, B, C$ to satisfy the choice axiom: the computations must resemble a plurality vote. For instance, to ensure that $P_{T}(A)=a, P_{T}(A)$ must be the sum of probabilities for the two rankings where $A$ is top ranked; i.e.,

$P_{T}(A)=a \frac{b}{1-a}+a \frac{c}{1-a}=a \frac{b+c}{1-a}=a$.

For $n \geqslant 3$ alternatives where $P\left(A_{j}\right)=a_{j}$, Eqs. (1.8), (3.1) generalize to

$R\left(A_{1} \succ \cdots \succ A_{n}\right)=a_{1} \times \frac{a_{2}}{1-a_{1}} \times \cdots \times \frac{a_{n-1}}{1-\sum_{1}^{n-2} a_{j}}$,

where to satisfy the choice axiom, Eq. (3.4) implicitly defines an unique way to compute probabilities: it is equivalent to using the plurality vote on each subset $S$.

As a brief aside, recall (Section 1.1) that the choice axiom does not require an universal set: alternatives can be added without renormalizing to make the sum of the probabilities unity. This feature is nicely demonstrated with Eq. (3.4) by observing that the transformation from $R(A \succ B \succ C)=a \frac{b}{1-a} \quad$ to $\quad R(A \succ B \succ C \succ D)=$ $\left[a \frac{b}{1-a}\right] \frac{c}{c+d}$ and $R(A \succ B \succ D \succ C)=\left[a \frac{b}{1-a}\right] \frac{d}{c+d}$ splits the original $a \frac{b}{1-a}$ value into two parts.

This description of Luce's choice of Eq. (3.1) suggests ways to generalize his notions. The alternative approach used here shows that

(1) Specifying the profile dictates how the $P_{T}(X)$ probabilities must be computed, but it imposes an overly severe restriction on the profiles. A much larger space of admissible profiles (for $n \geqslant 4$ alternatives), which provides added modelling flexibility, emerges by specifying only how to compute the probabilities. The admissible profiles are then implicitly defined by the computational decision rule and the choice axiom.

(2) There exists a continuum of new and natural ways to compute the $P_{T}(X)$ probabilities. This class, which can be identified with voting decision rules, provides new insights into the choice axiom.

\subsection{Implicitly defined profiles}

For the rest of this paper, after introducing an alternative way to compute the probabilities, the choice axiom is used to implicitly define all admissible profiles.

Definition 7. A Luce-Plurality probability decision rule for set $S$ is where the probability for alternative $A_{j}$ is the sum of the probabilities for each ranking in $S$ where $A_{j}$ is top-ranked. 

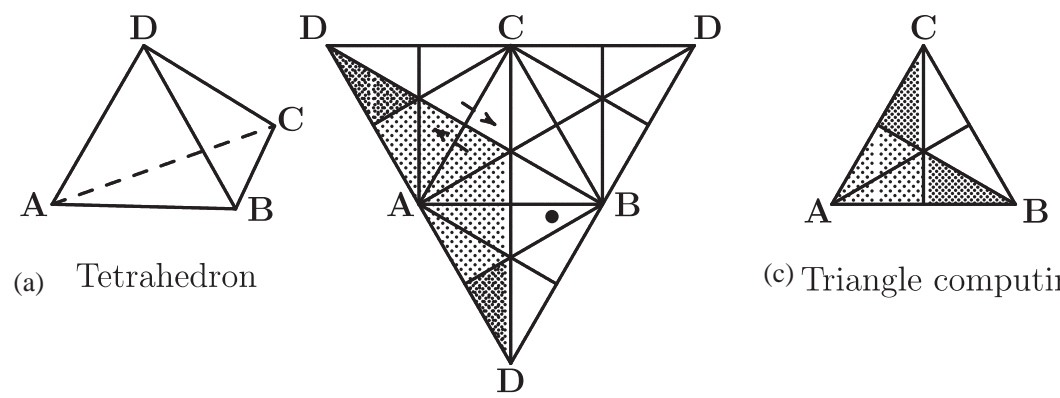

(c) Triangle computing

(b) Unfolded tetrahedron

Fig. 5. Representation triangle and tetrahedron: (a) tetrahedron, (b) unfolded tetrahedron and (c) triangle computing.

I call it the "Luce-Plurality rule" because this is the rule Luce uses and it is equivalent to the plurality vote. By implicitly defining the profiles, we discover many new ones. To explain with an analogy, consider the function $f(x, y)=\sqrt{x^{2}+y^{2}}$. If we concentrate on a specific domain point, such as $(3,4)$, we have that $f(3,4)=5$. But letting the outcome 5 define the supporting domain points, we have that $f^{-1}(5)$ is a circle of points that includes $(3,4)$ as a special case. Just as $f^{-1}(5)$ identifies a much larger set of supporting domain points, the approach of implicitly defining the profiles from the choice axiom identifies a significantly larger set of supporting probability profiles. Indeed, this implicit approach augments Eq. (3.4) profiles with so many new profiles that they must be described in terms of dimensional differences (or degrees of freedom).

Theorem 4. For $n=3$ alternatives, the only profiles satisfying the Luce-Plurality decision rule and the choice axiom with the standard pairwise computation form a two-dimensional manifold of $\mathscr{P}^{3}$; this profile space is parametrically described by Eq. (3.1).

For $n \geqslant 4$, the profiles satisfying the Luce-Plurality decision rule and the choice axiom (for the set of $n$ alternatives and all subsets) form $a(n-1)+[n !-$ $\left.2^{n-1}(n-2)-2\right]$ dimensional subspace of $\mathscr{P}^{n}$. This space is characterized by the $(n-1)$-dimensional space parameterized by Eq. (3.4) and a $\left[n !-2^{n-1}(n-2)-2\right]-$ dimensional "kernel" space of profiles.

The proof of this theorem is in Section 9

To indicate the sizable advantage offered by Theorem 4, while Luce's Eq. (3.4) restricts profiles to a $(n-1)$ dimensional submanifold of $\mathscr{P}^{n}$, the approach of implicitly defining profiles in terms of the computational method and the choice axiom allows a much richer profile space to emerge. With $n=5$ alternatives, for instance, Theorem 4 augments each Eq. (3.4) profile with a 70-dimensional subspace of profiles; i.e., rather than just a four-dimensional space of profiles, we have a 74-dimensional space of profiles that satisfy the choice axiom. When $n=6$, each profile in the original fivedimensional space defined by Luce's Eq. (3.4) has an associated 590-dimensional space of profiles with the same outcomes and properties. Then, with $n=10$, Eq. (3.4) defines a nine-dimensional space of profiles; the implicit approach attaches to each profile a much richer 3,624,704-dimensional profile subspace.

Now that we know there are other profiles that satisfy the choice axiom, the next task is to identify them. The larger class of $n=4$ profiles promised by Theorem 4 is described in terms of the Representation Tetrahedron (Saari, 2000a); this higher dimensional version of the representation triangle (Fig. 1) is used to represent fouralternative profiles. To convert this tetrahedron into a planar figure, choose a vertex ( $D$ in Fig. 5a) and cut along the three adjacent edges. By folding the three faces down (Fig. 5b), the 24 ranking regions are displayed in the flat figure. Again, the ranking associated with each region is determined by its distance to the vertices. For instance, the $\bullet$ is in the region closest to a $B$ vertex, next closest to $A$, then to $D$, and finally to $C$, so it represents $B \succ A \succ D \succ C$.

As in Fig. 1b, represent a profile by placing the ranking likelihood in the appropriate ranking region. The Luce-Plurality value for $P_{T}(A)$ is the sum of the regions where $A$ is top-ranked. The description for the three alternatives of $T=\{A, B, C\}$ was given earlier: recall that this computation sums the values in the two lightly shaded regions of Fig. 5c. Similarly, with the four alternatives $T=\{A, B, C, D\}, P_{T}(A)$ is the sum of values in the six lightly shaded regions of Fig. $5 \mathrm{~b}$. The LucePlurality value of $P_{S}(A)$ for $S=\{A, B, C\} \subset T$ is the sum all Fig. 5b ranking probabilities where $A$ is topranked among $\{A, B, C\}$ : it is the sum of the six regions in the lightly and the two more heavily shaded regions. (In the heavier shaded regions, the missing $D$ is topranked and $A$ is second-ranked.) For four alternatives, $P(A, B)$ is the sum of values in the 12 regions to the left of the vertical line in the flattened tetrahedron of Fig. 5b. Other $P_{S}(U)$ values for $T=\{A, B, C, D\}$ are determined in a symmetric manner. 


\subsection{Finding all $n=4$ profiles}

The proof of Theorem 4 (Section 9) has two parts. The first proves the known fact that any Eq. (3.4) profile satisfies the choice axiom. The second part comes from (Saari, 2000a) where it is shown that there exists a $\left[n !-2^{n-1}(n-2)-2\right]$-dimensional subspace of $\mathscr{P}^{n}$ where the profiles have no effect upon the plurality and pairwise tallies.

The $n=4$ profile changes, which augment the Eq. (3.4) probabilities and satisfy the choice axiom, are indicated by the two arrows in Fig. 5b. These arrows mean that after transferring the same probability value in the two directions among the four rankings, all plurality and pairwise tallies remain unchanged. These Fig. 5b arrows, then, represent where the Eq. (3.4) probabilities are altered by

$$
\begin{aligned}
& \mathscr{R}(A \succ C \succ B \succ D)=\frac{a c b}{(c+b+d)(b+d)}-\gamma_{A C}, \\
& \mathscr{R}(A \succ C \succ D \succ B)=\frac{a c d}{(c+d+b)(d+b)}+\gamma_{A C}, \\
& \mathscr{R}(C \succ A \succ D \succ D)=\frac{c a d}{(a+d+b)(d+b)}-\gamma_{A C}, \\
& \mathscr{R}(C \succ A \succ B \succ D)=\frac{c a b}{(a+b+d)(b+d)}+\gamma_{A C},
\end{aligned}
$$

where $\gamma_{A C}$ is any value, positive or negative, constrained only by the requirement that the resulting four ranking probabilities remain non-negative. The subscript indicates that the changes cross the tetrahedron edge connecting vertices $A$ and $C$. All possible kernel terms come from the six tetrahedron edges where values are symmetrically transferred in the same fashion; the choices from the six edges define the six extra dimensions of profiles promised by Theorem 4. By using the above geometric tallying process, a simple computation shows that such profile changes do not affect the probability values.

According to Theorem 4, each choice of $a, b, c, d$ values for the choice axiom generates a six-dimensional subspace of $\mathscr{P}^{4}$ rather than just the unique Eq. (3.4) profile. To determine this space, use the $a, b, c, d$ values to define a specific Eq. (3.4) four-alternative profile. The six-dimensional space of associated profiles (with identical probability outcomes for each set $S$ ) are obtained from the six $\gamma_{U V}$ variables associated with changes across the edge connecting vertex $U$ and $V, U, V=A, B, C, D$. The resulting values define a six-dimensional linear space constrained only by the requirement that each ranking probability is between zero and unity.

\section{Other probability decision rules}

Luce's claim that his definition of $P_{T}(X)$ is logically independent of the choice axiom suggests that there must exist other approaches. Later I will show that there exists a surprisingly wide spectrum of examples, but first a continuum of natural choices, motivated by the $\mathbf{w}_{s^{-}}$ positional voting methods, are described. View these probability measures as representing where a subject makes a distinction between the likelihood of selecting $A_{j}$ and the likelihood that $A_{j}$ is top-ranked. Namely, in computing $P_{T}\left(A_{j}\right)=a_{j}$-the probability that $A_{j}$ is most desirable - the subject goes beyond using the likelihood that $A_{j}$ is top-ranked to include information about where $A_{j}$ is second-ranked.

Definition 8. The three-alternative positional probability method $\mathbf{w}_{s}=(1, s, 0), 0 \leqslant s \leqslant 1$, is where $P_{T}^{s}\left(A_{j}\right)$ is the sum of the likelihoods of the two rankings where $A_{j}$ is topranked and $s$ times the sum from the two rankings where $A_{j}$ is middle ranked all divided by $(1+s)$. Call the three decision rules defined by $s=0, \frac{1}{2}, 1$, respectively, the Luce-Plurality method, the Borda method, and the antiplurality method.

Using Fig. 5c, compute $P_{T}^{s}(A)$ by adding an $s$-multiple of the sum of the entries in the two heavily shaded regions to the sum of the entries in the lighter shaded regions; the final sum is divided by $(1+s)$. The $P_{T}^{s}(A)$, $P_{T}^{s}(B), P_{T}^{s}(C)$ values for Fig. 1c are, respectively, $\frac{2+6 s}{10(1+s)}, \frac{5+2 s}{10(1+s)}, \frac{3+2 s}{10(1+s)}$. The term "antiplurality" for $\mathbf{w}_{1}$ reflects the decision rule's feature of equally weighting a first and second place alternative; it only distinguishes between the top two and the bottom-ranked alternative. As $\mathbf{w}_{1}$ identifies and penalizes a bottom-ranked alternative, we will investigate the role of $\mathbf{w}_{1}$ when developing profiles from "worst-first" instead of the "best-first" approach.

\subsection{Ranking probabilities}

When combined with the choice axiom, each $\mathbf{w}_{s}$-rule implicitly defines associated ranking probabilities. A convenient way to find these rankings and to associate them with the computational scheme is to expand the six rankings into a space of 12 rankings. Do so by dividing each ranking, say $A \succ B \succ C$, into a larger and smaller part of respective sizes $1 /(1+s)$ and $s /(1+s)$. Treat $P_{T}^{s}(A)$ as adding the larger portion of the two rankings where $A$ is top-ranked and the smaller portion of the two rankings where $A$ is middle-ranked. So, $\mathbf{w}_{1 / 4}=$ $\left(1, \frac{1}{4}, 0\right)$ divides the $j$ th ranking into a portion of size $1 /\left(1+\frac{1}{4}\right)=\frac{4}{5}$, with probability $\frac{4}{5} p_{j}$, and a smaller portion of size $\frac{1}{5}$, with probability $\frac{1}{5} p_{j}$. Computing $P_{T}^{1 / 4}(A)$ in this manner leads to

$P_{T}^{1 / 4}(A)=\frac{4}{5}\left(p_{1}+p_{2}\right)+\frac{1}{5}\left(p_{3}+p_{6}\right)$.

This division admits an interesting conditional probability interpretation that might roughly describe how a subject assembles ranking probabilities. To illustrate 


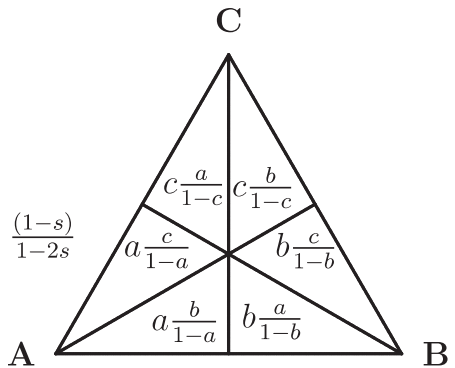

(a)

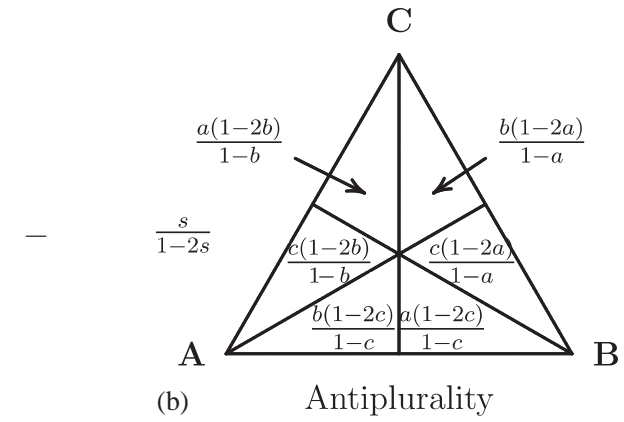

Fig. 6. Defining $\mathbf{w}_{s}=(1, s, 0)$ profiles: (a) plurality and antiplurality.

with $s=\frac{1}{4}$ and the type two ranking of $A \succ C \succ B$, the larger $\frac{1}{1+(1 / 4)} p_{2}=\frac{4}{5} p_{2}$ could represent the probability of selecting $A$ to be top-ranked given that $C$ is secondranked, while the smaller $\frac{1}{5} p_{2}$ is the likelihood of selecting $C$ as second-ranked given that $A$ is top-ranked. Thus, $p_{2}=\frac{4}{5} p_{2}+\frac{1}{5} p_{2}$ is the sum of probabilities of the two ways to assemble the $A \succ C \succ B$ ranking. In this fashion, $P_{T}^{1 / 4}(A)$ is the sum of the conditional probabilities where $A$ is top and second-ranked. Thus a $\mathbf{w}_{s^{-}}$ method captures the sense where, for a particular ranking say $A \succ C \succ B$, the ratio of [the likelihood $C$ is second-ranked given that $A$ is top-ranked] to [the likelihood $A$ is top-ranked given $C$ is second-ranked] is $s$.

As described in Theorem 5, each $\mathbf{w}_{s}$-rule determines a two-dimensional section of $\mathscr{P}^{3}$ profiles. Notice how this theorem significantly expands the applicability of the choice axiom by identifying a continuum of alternative ways to compute the probabilities. Also notice the difference between the plurality and antiplurality profiles: this difference will create reversal and other behaviors.

Theorem 5. For three alternatives and each $s \in$ $[0,1], s \neq \frac{1}{2}$, there is a uniquely defined, smooth twodimensional submanifold $\mathscr{M}_{s}$ of $\mathscr{P}^{3}$ profiles for which the $\mathbf{w}_{s}$-method satisfies the choice axiom. The ranking probability for ranking $\sigma$ is denoted by $\mathscr{R}^{s}(\sigma)$.

The space of probability profiles $\mathscr{M}_{1}$ for the antiplurality method is parameterized by

$\mathscr{R}^{1}(A \succ B \succ C)=b\left(2-\frac{1}{1-c}\right)=\frac{b(1-2 c)}{1-c}$

(where the five remaining probabilities are obtained by appropriate permutations of the names of the alternatives) for $\left\{(a, b, c) \mid a+b+c=1,0 \leqslant a, b, c \leqslant \frac{1}{2}\right\}$.

The $\mathscr{M}_{s}$ space of profiles associated with $\mathbf{w}_{s}, s \neq \frac{1}{2}$, are

$$
\begin{aligned}
\mathscr{R}^{s}(A \succ B \succ C)= & \frac{1-s}{1-2 s} \mathscr{R}^{0}(A \succ B \succ C) \\
& -\frac{s}{1-2 s} \mathscr{R}^{1}(A \succ B \succ C) .
\end{aligned}
$$

The only $\mathscr{P}^{3}$ profile that allows $P_{T}^{1 / 2}(X)$ (the Borda method with $\left.s=\frac{1}{2}\right)$ to satisfy the choice axiom with the standard pairwise probability computations is complete indifference $\left(\frac{1}{6}, \ldots, \frac{1}{6}\right)$.

Proof. Add the probabilities for the rankings in the plurality and antiplurality ways to determine that the pairwise and $P_{T}^{0}(X)$ and $P_{T}^{1}(X)$ outcomes are as specified. The $\mathbf{w}_{s}$ conclusions follow from the weighted sum definition of these profiles. The uniqueness assertion follows immediately by specifying the number of equations and unknowns. The assertions about the Borda method are described later.

Notice that Eq. (4.2) is not defined for $s=\frac{1}{2}$, the Borda method, and that the two coefficients change sign at this $s=\frac{1}{2}$ value. While this behavior can be explained in terms of the equations, a more satisfying explanation is given later and in Section 6 .

Eq. (4.2) profile is illustrated in Fig. 6. For a description, start with the $\mathscr{R}^{1}(\sigma)$ values illustrated in the triangle on the right-hand side of Fig. 6, and view the space as consisting of 12 , rather than six, rankings. The $P_{T}^{1}(X)$ computations divide each ranking into two equal parts; the $P_{T}^{1}(A)$ computation takes one of the two portions from each ranking where $A$ is not bottomranked. Thus the largest possible $P_{T}^{\mathrm{l}}(X)$ value is a half; this explains the $a \leqslant \frac{1}{2}$ upper bound.

As Fig. 6 shows that $\mathscr{R}^{s}(\sigma)$ is a weighted combination of the plurality, $\mathscr{R}^{0}(\sigma)$, and antiplurality, $\mathscr{R}^{1}(\sigma)$, ranking probabilities, a way to interpret $\mathscr{R}^{s}(\sigma)$ is to explain what the plurality and antiplurality ranking probabilities mean. Luce derives the plurality rankings from a "best-first" approach, so it remains to examine the $\mathscr{R}^{1}(\sigma)$ antiplurality computations. As the antiplurality emphasizes the bottom-ranked alternative (the only alternative not receiving points), it is reasonable to examine whether the $\mathscr{R}^{1}(\sigma)$ values admit an Eq. (1.9) "worst-first" scenario.

To start, the probability that $C$ is bottom-ranked is $p_{2}+p_{3}$ (the sum of ranking probabilities where $C$ is bottom-ranked, see Fig. 1a). To satisfy the choice axiom, the antiplurality outcome is $P_{T}^{1}(C)=\frac{1}{2}\left(p_{1}+p_{4}+\right.$ $\left.p_{5}+p_{6}\right)=c$. Combining these equations with $\sum_{j=1}^{6} p_{j}=$ 1 , it follows that the probability $C$ is bottom-ranked is $p_{2}+p_{3}=(1-2 c)$. The next Eq. (1.9) step is to compute 
the likelihood $B$ is bottom-ranked in $\{A, B\}$; according to Luce's $P(U, V)=P^{*}(V, U)$ condition, this is $1-$ $\frac{a}{a+b}=\frac{b}{a+b}=\frac{b}{(1-c)}$. Multiplying these two values in a Eq. (1.9) sense yields Fig. 6 and Theorem 5 value of

$\mathscr{R}^{1}(A \succ B \succ C)=\frac{b}{(1-c)}(1-2 c)$.

As anticipated, $\mathscr{R}^{1}(\sigma)$ admits a natural "worst-first" explanation.

This analysis means that the $\mathscr{R}^{s}(\sigma)$ probabilities of Fig. 6 can be viewed as determining ranking probabilities with a weighted combination of "best-first" and "worst-first" considerations - an interpretation that is compatible with the earlier "conditional probability" representation of assembling ranking probabilities. Moreover, since the $\mathscr{R}^{s}(A \succ B \succ C)$ value changes with $s$, different ways to compute the ranking probabilities lead to different values. In this manner, Theorem 5 generalizes the spirit of Luce's reversal result (Theorem 1) to a wider class of approaches and issues.

To conclude this subsection, the "worst-first" ranking probabilities for all $n \geqslant 3$ are described. As the antiplurality voting vector casts votes for all but the bottomranked alternative, a probability representation requires dividing each ranking into $(n-1)$ equal portions. The antiplurality outcome is the probability of the union of one portion from each ranking where the specified alternative is not bottom-ranked. Over subsets $S \subset T$, each ranking is divided into $|S|-1$ parts; divide each ranking into an appropriate number of portions where $1 /(|S|-1)$ of them are used for computations of set $S$. This leads to a representation with Eq. (3.4) flavor.

$$
\begin{aligned}
\mathscr{R}^{A P}\left(A_{1} \succ \cdots \succ A_{n}\right)= & \left(1-(n-1) a_{n}\right)\left[1-\frac{(n-2) a_{n-1}}{1-a_{n}}\right] \\
& \ldots\left[1-\frac{a_{2}}{1-\sum_{j=1}^{n-2} a_{j}}\right] .
\end{aligned}
$$

As with Theorem 4, attached to each Eq. (4.4) choice is a $\left[n !-2^{n-1}(n-2)-2\right]$ dimensional "kernel" space of profiles. All profiles from this larger space satisfy the choice axiom with the standard pairwise computation and the $P_{S}^{1}(X)$ computation for each set $S$.

Corollary 1. For $n \geqslant 3$ alternatives, Eqs. (3.4) and (4.4) define probability profiles that with, respectively, the Luce-Plurality and antiplurality methods satisfy the choice axiom. The first can be viewed as where the subject ranks alternatives from "superior choice first and then downwards," while the second represents profiles where a subject ranks them from "inferior choice first and then upwards." Attached to each profile is a $\left[n !-2^{n-1}(n-\right.$ $2)-2]$ dimensional subspace with the same properties.

If a subject computes $P_{T}(X)$ by using a combination of "best-first" and "worst-first" analyses, or if they uniformly include information about where alternative
$X$ is first and second ranked, then the above identifies the profiles and decision rules.

\subsection{Other consequences}

As a twist on reversal effects (with $n=3$ ), it is reasonable to wonder whether reversing a $\mathbf{w}_{s}$-probability profile reverses the $\mathbf{w}_{s}$-outcome. While this issue differs from Luce's Theorem 1, it is related in spirit. First, we need to provide a precise definition for "reversing a profile."

Definition 9. Let $\rho$ be the operation that reverses a ranking; e.g., $\rho(A \succ B \succ C)=C \succ B \succ A$. For $\mathbf{p}=$ $\left(p_{1}, p_{2}, p_{3}, p_{4}, p_{5}, p_{6}\right)$, let $\rho(\mathbf{p})=\left(p_{4}, p_{5}, p_{6}, p_{1}, p_{2}, p_{3}\right)$ be where the probability assigned to a ranking with $\mathbf{p}$ now is assigned to the reversed ranking in $\rho(\mathbf{p})$.

To develop intuition as to whether reversing a profile provides any useful relationships, experiment with $\mathbf{p}_{L}(a, b)$ to obtain

$\rho\left(\mathbf{p}_{L}(a, b)\right)=\left(\frac{c b}{1-c}, \frac{b c}{1-b}, \frac{b a}{1-b}, \frac{a b}{1-a}, \frac{a c}{1-a}, \frac{c a}{1-c}\right)$.

The Luce-Plurality outcome with $\mathbf{p}_{L}(a, b)$ is $P_{T}^{1}(A)=$ $a, P_{T}^{1}(B)=b, P_{T}^{1}(C)=c$ while the respective $\rho\left(\mathbf{p}_{L}(a, b)\right)$ values are $\frac{b c(1+a)}{(1-b)(1-c)}, \frac{a c(1+b)}{(1-a)(1-c)}, \frac{a b(1+c)}{(1-a)(1-b)}$. Beyond proving that if the $P_{T}^{0}$ ranking is $A \succ B \succ C$ for $\mathbf{p}_{L}$ then the $P_{T}^{0}$ ranking for $\rho\left(\mathbf{p}_{L}\right)$ is the reversed $C \succ B \succ A$, it seems unlikely to find more useful reversal relationships. ${ }^{1}$

A more complicated reversal relationship, however, does exist. To motivate the statement, because the antiplurality method emphasizes the bottom-ranked alternative it is, in a real sense, the reversal of the plurality method. After all, because $(1,1,0)=(1,1,1)-$ $(0,0,1)$, a way to compute $P_{T}^{1}(X)$ is to add the ranking probabilities where $X$ is bottom-ranked, subtract this value from 1 , and then normalize the values by dividing by 2 . This is equivalent to subtracting the $P_{T}^{0}(X)$ value of $\rho(\mathbf{p})$ from 1. (The value of " 1 " comes from the components of $(1,1,1)$.) Similarly, $\mathbf{w}_{1-s}$ can be viewed as the reversal of $\mathbf{w}_{s}$ : this follows because $(1,1-s, 0)=$ $(1,1,1)-(0, s, 1)$ where $(0, s, 1)$ refers to computing the $\mathbf{w}_{s}$-tally of $\rho(\mathbf{p})$.

The only difference for voting profiles is that the $\mathbf{w}_{s}$ tally for candidate $X$ for profile $\mathbf{p}$ can be obtained by subtracting $X$ 's $\mathbf{w}_{1-s}$ tally from $\rho(\mathbf{p})$ from $n$-the number of voters. To illustrate with Fig. $1 \mathrm{~b}$ profile, a quick way to find $\rho(\mathbf{p})$ is to interchange the values in diametrically opposite regions. Once this is done, the $\mathbf{w}_{1-s}$ tallies for $A, B$, and $C$ are, respectively, $6+$

\footnotetext{
${ }^{1}$ Notice that $\frac{b(1+a)}{(1-b)(1-c)}>\frac{a c(1+b)}{(1-a)(1-c)}$ if and only if $\frac{1-a^{2}}{a}>\frac{1-b^{2}}{b}$. Since $\frac{1-x^{2}}{x}$ is a strictly decreasing function, the $P_{T}^{0}$ rankings for $\mathbf{p}_{L}$ and $\rho\left(\mathbf{p}_{L}\right)$ always reverse each other. This relationship need not hold for other probability profiles nor for $P_{T}^{1}$.
} 
$12(1-s), 6+13(1-s)$, and $15+2(1-s)$. As $n=27$, we recover the $\mathbf{w}_{s}$ tallies in Fig. 1b of, respectively, $27-$ $[18-12 s]=9+12 s, 27-[19-13 s]=8+13 s, \quad$ and $27-[17-15 s]=10+15 s$.

Denote a profile's "tally" by $\tau^{s}(\mathbf{p})=\left(P_{T}^{s}(A)\right.$, $\left.P_{T}^{s}(B), P_{T}^{s}(C)\right)$. The following theorem states that an appropriate combination of these reversals always has tallies satisfying a precise numerical relationship; this relationship trivially extends to all $n \geqslant 3$ values.

Theorem 6. The following relationship among $P_{T}^{s}(X)$ values is satisfied for any probability profile $\mathbf{p}$ and $s \in[0,1]$.

$(1+s) \tau^{s}(\mathbf{p})+(1+(1-s)) \tau^{1-s}(\rho(\mathbf{p}))=(1,1,1)$.

Proof. It suffices to establish Eq. (4.5) for one alternative, say $A$. According to the definition, the $\mathbf{w}_{s}$ outcome for $\mathbf{p}$ is

$(1+s) P_{T}^{s}(A)=p_{1}+p_{2}+s p_{3}+s p_{6}$

while the $\mathbf{w}_{1-s}$ computation for $\rho(\mathbf{p})$ is

$(1+(1-s)) P_{T}^{1-s}(A)=p_{4}+p_{5}+(1-s) p_{3}+(1-s) p_{6}$.

The sum of the two expressions is $\sum_{j} p_{j}=1$; this proves the theorem.

It is worth emphasizing that Theorem 6 holds for any probability profile, not just those specified in Theorem 5 and Fig. 6. To suggest applications of Theorem 5, notice that since $\tau^{0}\left(\mathbf{p}_{L}(a, b)\right)=(a, b, c)$, we obtain $\tau^{1}\left(\rho\left(\mathbf{p}_{L}(a, b)\right)=\left(\frac{1}{2}(1-a), \frac{1}{2}(1-b), \frac{1}{2}(1-c)\right) \quad\right.$ Thus as $a>b>c$ creates a Luce-Plurality ranking of $A \succ B \succ$ $C$, the antiplurality ranking of the reversal of $\mathbf{p}$, of $\rho(\mathbf{p})$, is the opposite $C \succ B \succ A$. Similarly, the $P_{T}^{0}(X)$ values of the reversal of Fig. 6 profile $\mathbf{p}$ for $s=1$ must be $\tau^{0}(\rho(\mathbf{p}))=(1-2 a, 1-2 b, 1-2 c)$. In general, applying $\mathbf{w}_{s}$ to the reversal of Fig. 6 profile for $1-s$ leads to

$\tau^{1-s}(\mathbf{p})=(a, b, c)$,

$$
\begin{aligned}
\tau^{s}(\rho(\mathbf{p}))= & \frac{1}{1+s}(1-(2-s) a, 1-(2-s) b, \\
& 1-(2-s) c) .
\end{aligned}
$$

To illustrate with Fig. 1c probability profile that does not satisfy the choice axiom, $\tau^{0}(\mathbf{p})=\left(\frac{1}{5}, \frac{1}{2}, \frac{3}{10}\right)$, so $\tau^{1}(\rho(\mathbf{p}))=\left(\frac{2}{5}, \frac{1}{4}, \frac{7}{20}\right)$.

A lesson learned from Theorem 6 is that the nearly half-century long discussion about reversal effects and Theorem 1 may have concentrated on the wrong relationships. Instead, Eq. (4.5) offers an accurate reversal argument that holds for all probability profiles. Moreover, this expression goes beyond "best-first" and "worst-first" considerations to relate other reversal effects. More precisely, while we would like $\mathbf{w}_{s}$ and $\mathbf{w}_{1-s}$ to give the same ranking for a profile $\mathbf{p}$, we come close; $\mathbf{w}_{1-s}$ gives the reversed ranking to the reversed profile $\rho(\mathbf{p})$. (See Section 6.)
It is interesting to notice (Theorem 6) that only the Borda method $\left(s=\frac{1}{2}\right)$ admits a relationship between a profile and its reversal. (This follows because $\mathbf{w}_{1-s}=\mathbf{w}_{s}$ only for $s=\frac{1}{2}$.) This assertion seems to be unfortunate because, in general, the Borda method fails to satisfy the choice axiom with the standard pairwise computation (Theorem 5). But, as shown next, both this new reversal effect and the choice axiom are satisfied by using a nonstandard, nonlinear pairwise computation.

Definition 10. For $n=3$, let $P_{\text {Borda }}(A, B)=$ $\frac{2\left(p_{1}+p_{2}\right)+p_{3}+p_{6}}{1+2\left(p_{1}+p_{6}\right)+\left(p_{2}+p_{5}\right)}$. The numerator of $P_{\text {Borda }}(A, B)$ is the sum of ranking probabilities for the rankings where $A$ is middle ranked and twice the ranking probabilities where $A$ is top-ranked. The denominator is the sum of the numerators for $P_{\text {Borda }}(A, B)$ and $P_{\text {Borda }}(B, A)$.

$P_{\text {Borda }}(A, B)$ determines whether $A$ is more likely than $B$ by differentiating information about where $A$ is topranked from information where $A$ is second-ranked; $P_{\text {Borda }}(A, B)$ even uses information from the $B \succ A \succ C$ ranking where $B$ is ranked above $A$. In terms of Fig. 5c, $P_{\text {Borda }}(A, B)$ is the normalized value of twice the sum of the terms in the lightly shaded region (placing more value on where $A$ is top-ranked) plus the sum of the terms in the heavily shaded region (where $A$ is secondranked). What adds interest to this nonstandard pairwise computation is that with $P_{\text {Borda }}$, the Borda method satisfies the choice axiom for all probability profiles; there are no restrictions. While the LucePlurality approach holds only when restricted to a two-dimensional selection of probability profiles, the Borda method satisfies the choice axiom for all profiles.

Theorem 7. For $T=\{A, B, C\}$, if $P_{T}(X)$ is computed by using the Borda, $s=\frac{1}{2}$, method and pairwise probabilities are computed by using $P_{\text {Borda }}(U, V)$, then the system satisfies the choice axiom for all probability profiles.

The $P_{T}^{1 / 2}(X)$ values for a probability profile $\mathbf{p}$ and its reversal $\rho(\mathbf{p})$ are related in the following manner. For any $\mathbf{p}$, we have $\tau^{1 / 2}(\rho(\mathbf{p}))=\left(\frac{2}{3}, \frac{2}{3}, \frac{2}{3}\right)-\tau^{1 / 2}(\mathbf{p})$.

Proof. The first statement involves an elementary computation, the second is a direct consequence of Eq. (4.5).

The Borda system satisfies the choice axiom without needing to impose severe constraints on the choice of profiles, and it satisfies a reasonable reversal effect. From a mathematical perspective, this system is ideal for selecting ranking probabilities and using the choice axiom: it appears to be superior to the plurality approach. Whether the system models the behavior of subjects is a different, unexamined issue.

We encounter a mystery. Why does the Borda approach satisfy the choice axiom for all probability profiles while the other $\mathbf{w}_{s}$-methods are constrained to highly restricted choices? A "number of equations and 
number of unknowns" answer comes from comparing the information used to compute $P_{T}^{s}(A)$ and $P(A, B)$. With Fig. 1a, notice that $P_{T}^{0}(A)=p_{1}+p_{2}=a$ while $P(A, B)=p_{1}+p_{2}+p_{3}=a+p_{3}$ : the point to observe is that the two decision rules use different information. Consequently, without imposing the severe restriction that $p_{3}=\frac{a}{a+b}-a$, the choice axiom cannot be satisfied. Carrying this argument one step further, recall that the choice axiom requires $P(A, B)=\frac{P_{T}^{0}(A)}{P_{T}^{0}(A)+P_{T}^{0}(B)}$. With $T=$ $\{A, B, C\}$, the information needed to compute $P(A, B)$ is $p_{1}+p_{2}+p_{3}$. In contrast, the different $\frac{p_{1}+p_{2}}{\left(p_{1}+p_{2}\right)+\left(p_{5}+p_{6}\right)}$ information is needed for the right-hand side $\frac{P_{T}^{0}(A)}{P_{T}^{0}(A)+P_{T}^{0}(B)}$; one equation needs information about $p_{3}$ while the other requires information about $p_{5}$ and $p_{6}$. This radical difference in informational content means that very strong profile restrictions must be imposed to satisfy the choice axiom.

In other words, $P_{T}^{0}(A)$ uses information coming only from where $A$ is top-ranked while $P(A, B)$ uses this information and certain information about where $A$ is second ranked. As it is obvious, if two decision rules use different information, agreement (e.g., satisfying the choice axiom) occurs only by imposing coordinating constraints on the information: Fig. 6 constraints are mandatory.

This argument has other subtle messages. Because $P(A, B)$ uses information different from that used to compute $P_{T}^{0}(A), P_{T}^{0}(B)$, the standard pairwise computation violates $E q$. (1.5) spirit that the choice axiom attaches an intrinsic intensity to each alternative. In contrast, because the Borda method and $P_{\text {Borda }}(A, B)$ use the same information to compute outcomes, agreement holds for all profiles. In other words, $P_{\text {Borda }}$ and the Borda method appear to better capture the intrinsic intensity concept central to Luce's axiom. A similar argument holds for all $\mathbf{w}_{s}$-methods (where the numerator for $P(A, B)$ is $\left(p_{1}+\right.$ $\left.p_{2}\right)+s\left(p_{3}+p_{6}\right)$ and the denominator is the sum of the numerators for $P(A, B)$ and $P(B, A)$ ) when the same form of information is used for all computations, for different $S$, and for $n \geqslant 3$. A different explanation is in Section 6 .

To illustrate Theorem 7 statement about the Borda probabilities, since $\tau^{1 / 2}(\mathbf{p})=\frac{2}{3}(0.5,0.6,0.4)=\left(\frac{1}{3}, \frac{2}{5}, \frac{4}{15}\right)$ for Fig. 1c profile, $\tau^{1 / 2}(\rho(\mathbf{p}))=\frac{2}{3}(0.5,0.4,0.6)=\left(\frac{1}{3}, \frac{4}{15}, \frac{2}{5}\right)$. Observe the striking symmetry. To interpret this relationship, notice that $\left[P_{T}^{1 / 2}(X)-\frac{1}{3}\right]$ describes how the likelihood of selecting $X$ differs from the average likelihood $\left(\frac{1}{3}\right)$ of selecting an alternative. Since $\left[\frac{2}{3}-\right.$ $\left.P_{T}^{1 / 2}(X)\right]=\frac{1}{3}-\left[P_{T}^{1 / 2}(X)-\frac{1}{3}\right]$, the second statement asserts for any probability profile that the $\tau^{\frac{1}{2}}(\mathbf{p})$ and $\tau^{\frac{1}{2}}(\rho(\mathbf{p}))$ tallies are the same distance from the average $\left(\frac{1}{3}, \frac{1}{3}, \frac{1}{3}\right)$ values but reversing a profile reverses the sign.
These last comments provide an interpretation for Eq. (4.5). Dividing both sides of this equation by three shows that the $\tau^{s}(\mathbf{p})$ and $\tau^{1-s}(\rho(\mathbf{p}))$ tallies describe a balanced weighted difference relative to the average $\left(\frac{1}{3}, \frac{1}{3}, \frac{1}{3}\right)$ values.

\section{Comments about reversal}

The above comments about the Borda method suggest that maybe Theorem 1 can be understood in terms of informational differences in the computations of "best-first" and "worst-first" probability computations. To underscore the point, I include other ways to describe reversal conditions. With Theorem 5 profiles, for instance, we might wonder when the ranking probabilities associated with $\mathbf{w}_{0}$ and $\mathbf{w}_{1}$ agree: as described next, agreement holds only for complete indifference.

Corollary 2. For $n=3$, the $\mathbf{w}_{0}$ and $\mathbf{w}_{1}$ ranking probabilities agree if and only if $a=b=c=\frac{1}{3}$. More generally with the exception of the Borda method (i.e., for $\left.s_{1}, s_{2} \in\left[0, \frac{1}{2}\right) \cup\left(\frac{1}{2}, 1\right], s_{1} \neq s_{2}\right)$, the $\mathbf{w}_{s_{1}}$ and $\mathbf{w}_{s_{2}}$ values for their ranking probabilities agree if and only if $a=b=$ $c=\frac{1}{3}$. Thus the ranking probabilities agree if and only if all rankings have the same probability of $\frac{1}{6}$.

Proof. For the $\mathscr{R}^{s}(A \succ B \succ C)$ and $\mathscr{R}^{s}(B \succ A \succ C)$ values to agree for $s=0,1$, we need that $\frac{a b}{1-a}=\frac{b(1-2 c)}{1-c}$ and $\frac{b a}{1-b}=\frac{a(1-2 c)}{1-c}$. Cancelling $b$ from the first expression and $a$ from the second leads to

$\frac{a}{1-a}=\frac{1-2 c}{1-c}=\frac{b}{1-b}$.

The first and last terms require $a=b$. From the first two terms, $c=\frac{1-2 a}{2-3 a}=\frac{1-(a+b)}{2-3 a}=\frac{c}{2-3 a}$, or $a=\frac{1}{3}$. That is, $a=b=$ $c=\frac{1}{3}$. Substituting these values into Fig. 6 expressions force all ranking probabilities to equal $\frac{1}{6}$. The $s_{1} \neq s_{2}$ assertion follows from their different Fig. 6 weights.

While the above technical proof verifies the assertion, a more satisfying explanation comes from examining differences in the kind of information used by the different decision rules. For instance, $P_{T}^{0}(A)=p_{1}^{0}+p_{2}^{0}$ while $P_{T}^{1}(A)=p_{1}^{1}+p_{2}^{1}+p_{3}^{1}+p_{6}^{1}$ where $p_{j}^{s}$ is Fig. $6 j$ th ranking probability using the $s$ weight. Since $P_{T}^{1}$ goes beyond a $p_{1}^{1}+p_{2}^{1}$ tally to include $p_{3}^{1}+p_{6}^{1}$, the significant difference in sources of information requires us to anticipate agreement only in highly restrictive settings. Restating this comment in a mathematical perspective where different sources of information are interpreted as different variables, Corollary 5 must be anticipated because (as shown in Section 7) the pairwise probability computations required for the choice axiom define a four-dimensional subspace of $\mathscr{P}^{3}$ where, generically, two two-dimensional surfaces from $P^{0}$ and $P^{1}$ meet only in 
points: this intersection point is the profile of complete indifference.

\subsection{Various reversal approaches}

There are at least three ways to construct the "worstfirst" probability for the $A \succ B \succ C$ ranking.

(1) Luce introduces a second probability $P_{T}^{*}$ that satisfies his axiom to determine what it means for $C$ to be judged the inferior choice; this leads to Theorem 1.

(2) The antiplurality profile uses the likelihood that $C$ is bottom-ranked leading to Fig. 6 values for $s=1$ and Corollary 2. (The likelihood of being bottom-ranked need not be the likelihood of being judged the inferior choice.)

(3) A third approach does not change decision rules (as Luce does) by always using a specified decision rule. The probability $C$ is judged inferior is the probability that $C$ is judged superior for $\rho(\mathbf{p})$.

All three approaches lead to an extreme Corollary 2 type conclusion. To indicate why this happens with the last approach, first define a ranking probability using a "best-first" approach and a $\mathbf{w}_{s}$-system. For profile $\mathbf{p}=$ $\left(p_{1}, \ldots, p_{6}\right)$, the ranking probability for $A \succ B \succ C$, the $p_{1}$ value, must satisfy the $P_{T}^{s}(A) P(B, C)$ expression or

$p_{1}=\frac{1}{1+s}\left[p_{1}+p_{2}+s\left(p_{3}+p_{6}\right)\right]\left(p_{1}+p_{5}+p_{6}\right)$.

A similar expression holds for each $p_{j}$.

The "worst-first" outcome of $P^{*}(C) P^{*}(B, A)$, where $P_{T}^{*}(C)$ is the $P_{T}^{s}(C)$ value for $\rho(\mathbf{p})=\left(p_{4}, p_{5}, p_{6}, p_{1}, p_{2}, p_{3}\right)$ and $P^{*}(B, A)=P(A, B)$, defines the $p_{1}$ value equation

$p_{1}=\frac{1}{1+s}\left[p_{1}+p_{6}+s\left(p_{2}+p_{5}\right)\right]\left(p_{1}+p_{2}+p_{3}\right)$

with similar expressions for the other $p_{j}$ values. Although Eqs. (5.1), (5.2) are based on the same information (i.e., the same variables), the weight placed on this information is combined in different independent ways. Consequently, agreement can be expected only in restrictive settings. Stated mathematically, these expressions involve different, independent equations for the variables, so a "number of equations and unknowns" argument indicates that a solution is highly restricted. Indeed, it can be shown (with algebraic manipulations) that agreement holds only for the profile of complete indifference.

\subsection{Worst-first}

Rather than being surprised by disagreements between the "best-first" and "worst-first" ranking probabilities, we should be surprised if Theorem 1, or any of the various modifications offered here, allowed agreement with even one degree of freedom. After all, if two two-dimensional surfaces (one defined by the "bestfirst" approach and the other by the "worst-first" construction) in the four-dimensional subspace of $\mathscr{P}^{3}$ (required for the pairs to satisfy the choice axiom) intersect in a line, the expressions must have a strong degree of dependency; a dependency that might prove to be valuable.

To explain this dependency comment with more familiar geometry, consider a straight line and a twodimensional plane in $R^{3}$. If the line and plane never intersect, the line has the strong relationship of being parallel to the plane. If the straight line intersects the plane in several points, we have the stronger relationship that the line is in the plane; it is a special case of the plane. The general and expected situation is where the line meets the plane in a single point. Similarly, the general condition for two two-dimensional surfaces in the appropriate $\mathscr{P}^{3}$ subspace is to meet in a point (or, if not "flat" affine spaces, in isolated points). Consequently, if the surfaces meet in a line, they must enjoy a strong relationship; a relationship that might provide insight into individual decision making.

This extra relationship does not hold for the choice axiom; the only way $\mathscr{R}(\sigma)=\mathscr{R}^{*}(\sigma)$ agreement can be achieved over all $T=\{A, B, C\}$ rankings is with the probability profile of complete indifference. For $n=3$ this conclusion is due to Luce (1959), (Luce et al., 1965). Block and Marschak (1960) extended the result to all $n$. (Also see Yellott(1997a,b).) What makes this conclusion surprising, as reflected by the attention it has received, is that, intuitively, we expect the subject to use the same information to determine both $\mathscr{R}(\sigma)$ and $\mathscr{R}^{*}(\sigma)$, so both outcomes should agree. This is not the case; the following proof that $\mathscr{R}(\sigma)=\mathscr{R}^{*}(\sigma)$ requires "complete indifference" shows that different information is used with the two approaches. As demonstrated, the real source of the problem is a consequence of using the standard way to compute pairwise probabilities; e.g., these problems do not arise when the Borda method is used with $P_{\text {Borda }}$. Indeed, the source of the problem becomes apparent just by recognizing that $P(A) P(B, C)=\left(p_{1}+p_{2}\right)\left(p_{1}+p_{5}+p_{6}\right) \quad$ while $P^{*}(C) P(A, B)=\left(p_{1}+p_{6}\right)\left(p_{1}+p_{2}+p_{3}\right)$ : different information is used in each computation.

The first step in computing $\mathscr{R}^{*}(A \succ B \succ C)$ is to determine $P_{T}^{*}(C)$. A natural way to determine the likelihood that $C$ is judged the inferior alternative is to compute the probability that $C$ is the superior choice with $\rho\left(\mathbf{p}_{L}\right)$. This likelihood is

$$
\begin{aligned}
p_{1}+p_{6} & =\mathscr{R}(A \succ B \succ C)+\mathscr{R}(B \succ A \succ C) \\
& =a \frac{b}{a+b}+b \frac{a}{a+b} .
\end{aligned}
$$


Luce shows that the $P_{T}^{*}$ value is different; it is (see Luce, 1959, Theorem 1, p. 16)

$P_{T}^{*}(C)=\frac{a b}{a b+a c+b c}=\frac{\frac{1}{c}}{\frac{1}{a}+\frac{1}{b}+\frac{1}{c}}$.

This Eq. (5.4) value dictates the complete indifference conclusion, so it is important to understand, beyond algebraic computations, why it arises.

While the $P_{T}^{*}(C)$ computation is the $p_{1}+p_{6}$ sum, the reason Eq. (5.4) value occurs is for $P^{*}$ to satisfy the choice axiom. Namely, these $p_{j}$ terms have different values; call them $p_{j}^{*}$. The source of these different $p_{j}^{*}$ values comes from the fact that $P^{*}(C)=p_{1}^{*}+p_{6}^{*}=c^{*}$ while $P^{*}(C, B)=p_{1}^{*}+p_{6}^{*}+p_{5}^{*}=\frac{c^{*}}{b^{*}+c^{*}}$ where, with the $P(B, C)=P^{*}(C, B)$ assumption, $P^{*}(C, B)=\frac{b}{b+c}$. In other words, $P^{*}(C, B)$ uses more information than $P^{*}(C)$, so a strict constraint must be imposed on the $p_{5}^{*}$ value. (By symmetry, a constraint is imposed on all $p_{i}^{*}$ values.) The $P(B, C)=P^{*}(C, B)$ assumption relates the $x$ and $x^{*}$ values, $x=a, b, c$. Compare this source of $p_{5}^{*}$ with the very different source of information defining $p_{5}$; e.g., $p_{5}=P(C, A)-P_{T}(C)$. The following result (particularly Eq. (5.5)), shows that this interpretation of the $\mathscr{R}^{*}(\sigma)$ values is consistent with Eq. (5.4).

Proposition 3. For $n=3$, assume $P_{T}$ and $P_{T}^{*}$ are defined over $T$ and its subsets with imperfect discrimination. Suppose $P_{T}$ satisfies the choice axiom, and that $P_{S}^{*}\left(A_{j}\right)$ is the probability that $A_{j}$ is judged inferior in $S$. Assume that $P^{*}(B, A)=P(A, B)$ and that $P_{T}^{*}$ satisfies the choice axiom. If $R^{*}(A \succ B \succ C)=P_{T}^{*}(C) P^{*}(B, A)$, we have that

$$
\begin{aligned}
R^{*}(A \succ B \succ C) & =\left[\frac{a}{a+b}\right]\left[\frac{a b}{a b+a c+b c}\right] \\
& =P^{*}(B, A)-P_{T}^{*}(B) .
\end{aligned}
$$

With the same assumptions for $n \geqslant 3$, the probability profile is given by

$R^{*}\left(A_{1} \succ \cdots \succ A_{n}\right)=\prod_{j=2}^{n} P_{S_{j}}^{*}\left(A_{j}\right)$

where $S_{j}$ consists of the top $j$ ranked alternatives $\left\{A_{1}, \ldots, A_{j}\right\}$ and

$P_{S_{j}}^{*}\left(A_{j}\right)=\frac{a_{1} a_{2} \cdots a_{j-1}}{\sum_{i=1}^{j}\left(a_{1} a_{2} a_{3} \cdots a_{j} / a_{i}\right)}=\frac{\frac{1}{a_{j}}}{\sum_{i=1}^{j} \frac{1}{a_{i}}}$.

Proof. The product representations are direct consequences of Luce (1959, Theorem 1, p. 16). The subtraction representation follows from Fig. 1 where $B$ is bottom-ranked only with type two and three rankings, so $P_{T}^{*}(B)=p_{2}^{*}+p_{3}^{*}$. But since $P^{*}(B, A)(=P(A, B))=$ $p_{1}^{*}+p_{2}^{*}+p_{3}^{*}$, we have that $p_{1}^{*}=R^{*}(A \succ B \succ C)=$ $P^{*}(B, A)-P_{T}^{*}(B)$. To check whether this geometric representation agrees with the assumptions, substitute $P^{*}(B)=\frac{a c}{a b+b c+a c}$ and $P(A, B)=\frac{a}{(a+b)}$ into the second expression; algebraic computations prove that, with imperfect discrimination, equality holds. (By use of Fig. 4, a related, more complicated expression follows for $n=4$.)

This difference in the kind of information used to define $p_{j}$ and $p_{j}^{*}$ imposes a strong constraint in order to achieve agreement.

Proposition 4. Using the assumptions of Theorem 1, a necessary and sufficient condition for $R(A \succ B \succ C)=$ $R^{*}\left(A \succ^{*} B \succ^{*} C\right)$ is $P(A, B)=P(B, C)$ or

$P(B, C)-P_{T}(B)=P(A, B)-P_{T}^{*}(B)$.

Moreover, $P(B, C)=P(A, B)$ if and only if $P_{T}^{*}(B)=$ $P_{T}(B)$.

In words, for ranking probabilities to agree whether computed top-down or bottom-up, a necessary and sufficient condition is that the $P_{T}$ likelihood of the middle ranked alternative being the superior choice equals its $P_{T}^{*}$ likelihood of being the inferior choice.

Proof. With imperfect discrimination, $P(A, B)=$ $P(B, C)$ if and only if $a c=b^{2}$. But $R(A \succ B \succ C)=$ $R^{*}\left(A \succ^{*} B \succ^{*} C\right)$ if and only if

$\frac{a b}{(b+c)}=\left(\frac{a}{a+b}\right)\left(\frac{a b}{a b+a c+b c}\right)$.

By cross multiplying and collecting terms, equality holds iff $a b(1-a-b-c)=c\left(b^{2}-a c\right)$. As the lefthand side is zero, it follows from imperfect discrimination that equality holds iff $b^{2}=a c$. This proves the assertion.

Eq. (5.7) follows from the $p_{1}$ and $p_{1}^{*}$ representations. To prove that $P_{T}^{*}(B)=b$ iff $P(A, B)=P(B, C)$, notice that $P_{T}^{*}(B)=b$ iff $a c=b(a b+a c+b c)$, or $a c(1-b)=$ $b^{2}(1-b)$. With imperfect discrimination, equality holds iff $a c=b^{2}$; this proves the assertion.

As an alternative is middle ranked in precisely two strict rankings, the "worse-first" and "best-first" surfaces agree along the three lines $P^{*}(X)=P(X), X=$ $A, B, C$. But these lines of agreement intersect only at the point of complete indifference, so the only way all ranking probabilities agree, whether computed from top down or bottom up, is with the profile of complete indifference. This is Luce's (1959) conclusion; also see Yellott(1997a,b). Namely, the combination of the standard way to compute pairwise probabilities with the choice axiom force very different information to be used when computing $\mathscr{R}(\sigma)$ and $\mathscr{R}^{*}(\sigma)$; these differences permit agreement only with complete indifference.

Theorem 8. Under the assumptions of Theorem $1, p_{j}=p_{j}^{*}$ for all $j=1, \ldots, 6$, iff $P^{*}(A)=a, P_{T}^{*}(B)=b, P_{T}^{*}(C)=c$. This condition, which requires $a=b=c=\frac{1}{3}$, requires the 
profile to be of complete indifference where each ranking probability is $\frac{1}{6}$.

Proof. The equivalence in the first statement follows from the above discussion. According to the above, $P_{T}^{*}(B)=b$ iff $P(A, B)=P(B, C)$ iff $p_{1}+p_{2}+p_{3}=p_{1}+p_{5}+p_{6}$ iff $p_{2}+p_{3}=p_{5}+p_{6}$ iff $p_{2}^{*}+p_{3}^{*}=p_{5}^{*}+p_{6}^{*}$ iff $P_{T}^{*}(B)=P_{T}^{*}(C)$ iff $b=c$. The $a=b$ equality is found similarly. The fact that the profile is one of complete indifference follows either from the form of $\mathbf{p}_{L}(a, b)$ or by solving the three equations for $P_{T}(X)=\frac{1}{3}$ and the expressions $P(A, B)=$ $P(B, C)=P(A, C)=\frac{1}{2}$ for the $p_{j}$ values.

\section{Explanations and the Ranking Axiom}

To answer some of the remaining mysteries we have encountered, I use the earlier (Section 2) results from the positional and pairwise voting literature.

\subsection{Choice Axiom}

Theorem 3 shows that all conflict among outcomes of the pairs and the triplet disappears by restricting attention to the basic profiles, or by using Borda's method and restricting profiles so that they have no Condorcet term. Alternatively, we could search for a judicious combination of Condorcet and reversal profiles that alters the pairwise outcomes in just the correct amount to agree with the adjusted $P_{T}^{0}(X)$ values of the triplet. This last scenario characterizes precisely what happens with the choice axiom and Luce's profile of Eqs. (3.2), (3.4) as well as with all the profiles of Theorem 4, Fig. 6.

All conflict due to the pairwise vote and tallies is caused by the $\mathbf{p}_{\text {Con }}$ term (which does not affect $\mathbf{w}_{s^{-}}$ outcomes). This component causes all cycles and nontransitive pairwise rankings, all differences among methods using pairwise votes, all differences between the tallies of the pairwise majority votes and other voting rules and so forth. The explanation, as developed in Saari $(1999,2000 a, b)$ is that the Condorcet term can be viewed as replacing this portion of transitive preferences with cyclic preferences.

As this last observation explains properties of the choice axiom, it is worth being more explicit. The Condorcet triplet $A \succ B \succ C, B \succ C \succ A, C \succ A \succ B$ creates a cycle manifested here by two $A \succ B$ and one $B \succ A$ rankings, two $B \succ C$ and one $C \succ B$ rankings, and two $C \succ A$ and one $A \succ C$ rankings. But the same sequence of pairwise rankings results from profiles that violate transitivity by having cyclic preferences. (Two choices, for instance, are the $A \succ B \succ C, C \succ B \succ$ $A$ and $A \succ B, B \succ C, C \succ A$ preferences and the two $A \succ B, B \succ C, C \succ A$ cyclic choices with one $B \succ A, C \succ B, A \succ B$.) Even if transitive preferences are intended, it can be shown that the decision rule interprets the data as coming from cyclic preferences. ${ }^{2}$ In particular, the Condorcet term causes all problems; indeed, if profiles have no Condorcet terms, then Arrow's conditions are satisfied by the Borda method rather than just a dictator. (Also see Saari \& Sieberg, 2001.)

With probability profiles, the Condorcet term reflects a subject's cyclic ambiguity in ranking the alternatives. It is interesting how the choice axiom implicitly mandates this ambiguity when the standard pairwise computation is used.

The Condorcet term only affects $P(A, B)$ values, so other profile components must be introduced to alter the $P_{T}^{s}(X)$ values to achieve Luce's numeric precision. These are the $\mathbf{p}_{\mathrm{Rev}}$ terms that have no influence on pairwise or Borda outcomes, but they create differences among $P_{T}^{s}(X)$ outcomes (for $s \neq \frac{1}{2}$ ). Theorem 5 ranking profiles, then, require a precise level of $\mathbf{p}_{\mathrm{Rev}}$ components to correspond with the profile's Condorcet component. It is this needed precision among reversal and Condorcet components that imposes the restrictions on the profiles that satisfy the choice axiom.

Recall from (2) of Theorem 3 that this $\mathbf{p}_{\text {Rev }}$ term has an opposing effect on the Luce-Plurality computation (which emphasizes the top-ranked alternative) as with the anti-plurality computation (which emphasizes the bottom-ranked alternative). Thus, the larger the magnitude of this $\mathbf{p}_{\mathrm{Rev}}$ term relative to $\mathbf{p}_{\mathrm{Ba}}$, the greater the difference when emphasizing the best or the worst alternative. As different $P_{T}^{s}$ computations have different $\mathbf{p}_{\text {Rev }}$ outcomes, a different component level of the Condorcet term is needed to coordinate the $P_{T}^{s}(X)$ and $P(A, B)$ values to satisfy the choice axiom; the phrase "compensating errors" is a way to describe this affect of adding components that should cancel. This description, where different amounts of profile differentials are needed for different $s$ values, shows that the ranking probabilities can be expected to coincide only when the Condorcet and $\mathbf{p}_{\mathrm{Rev}}$ values are zero: this is the profile of complete indifference.

\subsection{Borda properties}

Other remaining mysteries are to explain why the Borda $P_{T}^{1 / 2}(X)$ is excluded from Theorem 5 and why Eq. (4.2) coefficients approach infinity as $s \rightarrow \frac{1}{2}$. To explain, since the Condorcet component of a profile affects the $P(A, B)$ values, something must be done to appropriately adjust the Borda $P_{T}^{1 / 2}(X)$ values. But Theorem 3 proves this is impossible because reversal and Condorcet terms do not effect the Borda outcome. Similarly, as $s \rightarrow \frac{1}{2}$, it follows from Theorem 3 that the $P_{T}^{s}$ value for $\mathbf{p}_{\text {Rev }}$ must approach zero. To compensate, larger amounts of $\mathbf{p}_{\mathrm{Rev}}$ is

\footnotetext{
${ }^{2}$ This conflict between actual preferences and the way a decision rule must "interpret" them explains Arrow's Theorem and suggests ways to circumvent his negative assertion (Saari, 2001a,b).
} 
needed. But as $s$ passes through $\frac{1}{2}$, the reversal terms has a reversed effect on the $P_{T}^{s}(X)$ ranking, so the Condorcet term needs to be reversed; the coefficients of Eq. (4.2) reverse sign.

The interesting fact is that by replacing the standard pairwise computation with $P_{\text {Borda }}(A, B)$, all of the difficulties with the Borda method disappear (Theorem 7). As explained next, $P_{\text {Borda }}(A, B)$ is not affected by Condorcet terms, so there is no need to adjust $P_{T}^{1 / 2}(X)$ values.

Theorem 9. For $n=3$ alternatives, the $P_{\text {Borda }}(U, V)$ outcome of any reversal or Condorcet component for any pair is zero. The only terms in a profile that affects the $P_{\mathrm{Borda}}(U, V)$ outcome are the neutral $\mathbf{p}_{\mathrm{Neu}}$ and basic $\mathbf{p}_{\mathrm{Ba}}$ components.

Proof. This is a direct computation; e.g., use Fig. 3.

\subsection{Decompositions}

A way to underscore the above comments about the need for coordination among the components is to identify the $\mathbf{p}_{\text {Rev }}$ and $\mathbf{p}_{\text {Con }}$ components in Theorem 5 profiles. The next theorem computes this decomposition for any probability profile; the result is then applied to determine the decomposition of Theorem 5 profiles.

Theorem 10 (Saari, 1999). By vector addition, a profile expressed in the

$\mathbf{p}=a_{B} \mathbf{B}_{A}+b_{B} \mathbf{B}_{B}+a_{R} \mathbf{R}_{A}+b_{R} \mathbf{R}_{B}+\gamma \mathbf{C}^{3}+k \mathbf{K}$

form can be written in the $\mathbf{p}=\left(p_{1}, \ldots, p_{6}\right)$ format. Conversely, for a given probability profile $\mathbf{p}=$ $\left(p_{1}, \ldots, p_{6}\right)$, the vector $\mathbf{v}=\left(a_{B}, b_{B}, a_{R}, b_{R}, \gamma, k\right)$ of coefficients of Eq. (6.1) profile decomposition are obtained from the matrix expression $\mathbf{v}=T(\mathbf{p})$ where

$T=\frac{1}{6}\left(\begin{array}{cccccc}2 & 1 & -1 & -2 & -1 & 1 \\ 1 & -1 & -2 & -1 & 1 & 2 \\ 0 & 1 & -1 & 0 & 1 & -1 \\ -1 & 1 & 0 & -1 & 1 & 0 \\ 1 & -1 & 1 & -1 & 1 & -1 \\ 1 & 1 & 1 & 1 & 1 & 1\end{array}\right)$.

Proof. Expressing Eq. (6.1) in a matrix representation $\mathbf{p}=\mathscr{A}(\mathbf{v})$, we have that matrix $T=\mathscr{A}^{-1}$ converts profile $\mathbf{p}$ into its profile decomposition format.

The decomposition of Theorem 5 profiles, determined by using matrix $T$, follows.

Theorem 11. The profile decomposition for the LucePlurality profiles is

(1) $a_{B}=\frac{1}{6}\left[\frac{a b}{b+c}+a-c-\frac{b c}{a+b}+-b \frac{a-c}{a+c}\right]$,

(2) $b_{B}=\frac{1}{6}\left[a \frac{b-c}{b+c}-c-\frac{a c}{a+b}+b+\frac{b a}{a+c}\right]$,

(3) $a_{R}=\frac{1}{6}\left[a c\left[\frac{1}{b+c}-\frac{1}{a+b}\right]+b \frac{c-a}{c+a}\right]$,
(4) $b_{R}=\frac{1}{6}\left[a \frac{c-b}{b+c}+b c\left[\frac{1}{a+c}-\frac{1}{a+b}\right]\right.$,

(5) $\gamma=\frac{1}{6}\left[\frac{c(a-b)}{a+b}+\frac{a(b-c)}{b+c}+\frac{b(c-a)}{c+a}\right]$.

The decomposition for the Antiplurality profiles is

(1) $a_{B}=\frac{1}{6}\left[\frac{(2 b+a)(1-2 c)}{a+b}+\frac{(c-a)(1-2 b)}{a+c}-\frac{(2 b+c)(1-2 a)}{b+c}\right]$,

(2) $b_{B}=\frac{1}{6}\left[\frac{(a+2 b)(1-2 c)}{a+b}-\frac{(2 a+c)(1-2 b)}{a+c}+\frac{(c-b)(1-2 a)}{b+c}\right]$,

(3) $a_{R}=\frac{1}{6}\left[-\frac{a(1-2 c)}{a+b}+\frac{(c-a)(1-2 b)}{a+c}-\frac{c(1-2 a)}{b+c}\right]$,

(4) $b_{R}=\frac{1}{6}\left[-\frac{b(1-2 c)}{a+b}+\frac{c(1-2 b)}{a+c}+\frac{(c-b)(1-2 a)}{b+c}\right]$,

(5) $\gamma=\frac{1}{6}\left[\frac{(b-a)(1-2 c)}{a+b}+\frac{(a-c)(1-2 b)}{a+c}+\frac{(c-b)(1-2 a)}{b+c}\right]$.

The decomposition of the $\mathbf{w}_{s}$ profiles is $(1-s) /(1-2 s)$ times the Plurality term minus $s /(1-2 s)$ times the Antiplurality term.

Proof. This is $T(\mathbf{p})$ where $\mathbf{p}$ has a Eq. (1.8) representation.

Notice the careful coordination between Reversal and Condorcet terms. For instance, since $a+b=1-c$, we see from the denominators of $a_{R}$ and $\gamma$ how, as $a$ or $a+b$ approach unity, the terms become quite dominant. Also, by experimenting with different $a, b$ values, one can see the different signs of the reversal and Condorcet terms for $s=0,1$. The careful coordination needed to achieve the numerical precision required by the choice axiom is fascinating.

An alternative way to use $\mathbf{w}_{s}$ methods to define ranking approaches is to find a profile decomposition of Theorem 3 form that emphasizes the $\mathbf{w}_{s}$ voting rule. This program has been carried out (Saari, 2002): for convenience, definitions are given.

Definition 11 (Saari, 2002). For a specified $\mathbf{w}_{s}$ computation method, $0 \leqslant s \leqslant 1$, an $A_{j}$-Basic profile differential, $\mathbf{B}_{A_{j}}^{s}$, assigns $2-s$ points to each ranking where $A_{j}$ is topranked, $2 s-1$ points to each ranking where $A_{j}$ is middle ranked, and $-(1+s)$ points to each ranking where $A_{j}$ is bottom-ranked. The $\mathbf{w}_{s}$ Basic profile space, consisting of all Basic profile differentials, is the $\mathscr{T}^{3}{ }^{3}$ subspace spanned by $\left\{\mathbf{B}_{A_{j}}^{s}\right\}_{j=1}^{3}$.

The $A_{j}$-Orthogonal profile differential, $\mathbf{O}_{A_{j}}^{s}$, assigns $-s$ points to each ranking where $A_{j}$ is top ranked, 1 point where $A_{j}$ is middle ranked, and $-1+s$ points to each ranking where $A_{j}$ is bottom-ranked. The $\mathbf{w}_{s}$ Orthogonal subspace, consisting of all Orthogonal profile differentials, is the $\mathscr{T} \mathscr{P}^{3}$ subspace spanned by $\left\{\mathbf{O}_{A_{j}}^{3}\right\}_{j=1}^{3}$.

Only the $\mathbf{w}_{s}$ Basic terms affect a $\mathbf{w}_{s}$ computation (Saari, 2002). So, for the Luce-Plurality method, the space of profiles given by

$$
\begin{aligned}
\mathbf{p}= & a_{B}^{0}(2,2,-1,-1,-1,-1)+b_{B}^{0}(-1,-1,-1,-1,2,2) \\
& +k(1,1,1,1,1,1)
\end{aligned}
$$




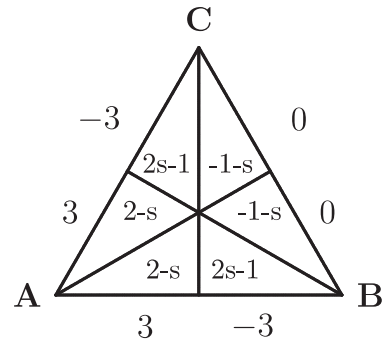

(a) $\quad \mathbf{w}_{s}$ Basic for $\mathrm{A}$

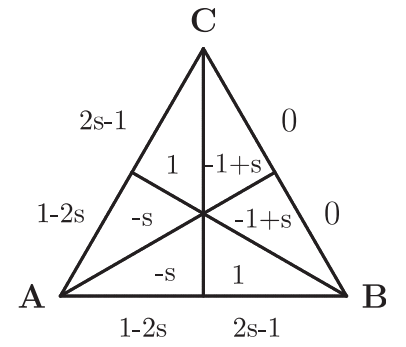

(b) $\mathbf{w}_{\mathbf{s}}$ Orthogonal for $\mathrm{A}$
Fig. 7. $\mathbf{w}_{s}$ standard profiles: (a) $\mathbf{w}_{s}$ basic for A and (b) $\mathbf{w}_{s}$ orthogonal for A.

describes all outcomes; terms in the Condorcet and Orthogonal direction affect, respectively, pairwise and other $\mathbf{w}_{s}$ and pairwise computations (Fig. 7). What is interesting, and relates to the reversal concerns, is that while the Luce-Plurality method is not affected by any profile components in the $\mathbf{w}_{0}$ Orthogonal directions of $(0,0,1,-1,-1,1)$ and $(1,-1,-1,1,0,0)$, these profile components do affect all other $\mathbf{w}_{s}$ methods. This conclusion captures, in another sense, our earlier arguments.

It remains to briefly discuss the Ranking Axiom of Section 1. A quick approach is to notice that the main difference from the choice axiom is that Ranking Axiom foregoes numerical precision for consistency in rankings. Consequently, it follows from continuity considerations that for each $\mathbf{w}_{s}$ method there is an open set surrounding the manifold of its Fig. 6 profiles where the ranking axiom is satisfied but the choice axiom is not. In fact, with the profile decomposition, it is possible to describe this region in terms of simple algebra by using an approach similar to that given in Saari (1999) to describe when all voting paradoxes occur. The advantage is obvious; the conditions now become robust in that they hold for a much wider selection of probability profiles. Also, as described above, in a neighborhood about the basic profiles, the Ranking Axiom holds while Luce's does not. This establishes the existence of a continuum of examples that satisfy the Ranking but not the choice axiom.

\section{Generalizations and a binary decomposition}

It remains to examine more closely the binary probabilities and to show that many of the above results can be generalized. Again, Fig. 1 geometric profile representation is used. As an aside to show how this approach facilitates computations, recall from Fig. 1a that the standard $P(B, A)$ computation (Definition 3, Eq. (1.16)) is the sum of the probabilities of the three rankings in the shaded region; i.e., $P(B, A)=$ $p_{4}+p_{5}+p_{6}$. To find all profiles satisfying Debreu's three pairwise conditions where the sum of each pairwise probability is $\frac{1}{2}$, place $x, y$, and $z$ in each of two diametrically opposing ranking regions. (So, we are using the reversal configurations of Section 2.) Since all choices of these values require $x+y+z=\frac{1}{2}$, any choice from this continuum suffices.

\subsection{Choice profile sets}

To implicitly define the profiles and decision rules that satisfy the choice axiom, it is important to describe the restrictions that are imposed on the admissible profiles when its condition (2) is applied only to pairs of alternatives. ${ }^{3}$ The next definition emphasizes the consequences of the choice axiom for binaries. As we have seen, further restrictions arise by applying Luce's conditions to $|S|=j, j=3, \ldots, n$.

Definition 12. Let the choice binary outcomes for the $n$ alternatives $\left\{A_{1}, \ldots, A_{n}\right\}$ be where there exist $n$ positive scalars $\left\{a_{1}, \ldots, a_{n}\right\}, \sum_{j=1}^{n} a_{j}=1$, so that for all $i \neq j$, $P\left(A_{i}, A_{j}\right)=a_{i} /\left(a_{i}+a_{j}\right)$. In the $(n !-1)$-dimensional profile space $\mathscr{P}^{n}$, the choice profile set, denoted by $\mathscr{C} \mathscr{B}^{n}$, is the set of all profiles $\mathbf{p}$ where the pairwise outcomes under a specified computation are choice binary outcomes.

To explain this definition, the choice axiom requires $a_{i} /\left(a_{i}+a_{j}\right)$ values for the pairwise computations for choices of $a_{j}$. But the manner in which the profiles are combined to determine these values can vary. So, the "specified computation" refers to how to compute pairwise probabilities. Space $\mathscr{C} \mathscr{B}^{n}$ are those profiles where, with the specified computation of pairwise probabilities, the probabilities have the required form.

To verify that $\mathscr{C} \mathscr{B}^{3}$ restricts profiles, notice that Fig. 1c profile is not in $\mathscr{C} \mathscr{B}^{3}$. If it were, the standard pairwise computation giving its $A \sim B$ and $A \sim C$ tied outcomes would require, from Eq. (1.7), that $P(B, C)=\frac{1}{2}$ : this value contradicts the $\frac{7}{10}$ to $\frac{3}{10}$ dominance of $B$ over $C$. So, a way to understand the choice axiom is to determine the $\mathscr{C} \mathscr{B}^{n}$ structure. (Also see Fishburn, 2002.) The following result is stated for the standard pairwise computation, but it holds for any linear pairwise computation that includes an open set of choice binary outcomes.

Theorem 12. For $n \geqslant 2$ alternatives and the standard pairwise computation, $\mathscr{C} \mathscr{B}^{n}$ is a smooth submanifold of profile space $\mathscr{P}^{n}$ with codimension $\left(\begin{array}{l}n \\ 2\end{array}\right)-(n-1)$.

For three alternatives, $\mathscr{C} \mathscr{B}^{3}$ is a codimension $\left(\begin{array}{l}3 \\ 2\end{array}\right)-$ $(3-1)=1$ submanifold. By excluding only one dimension, $\mathscr{C} \mathscr{B}^{3}$ is a four-dimensional surface in the

\footnotetext{
${ }^{3}$ Logically, this description should precede Section 3, and it did in an earlier version. But following a referee's suggestion, the material was reorganized to make the paper easier to follow.
} 
five-dimensional profile space $\mathscr{P}^{3}$. As already shown, profiles satisfying $\mathbf{w}_{s}$-outcomes and the choice axiom form a two-dimensional section of this four-dimensional space. For five alternatives, $\mathscr{C} \mathscr{B}^{5}$ is a $[5 !-1]-\left[\left(\begin{array}{l}5 \\ 2\end{array}\right)-(5-1)\right]=$ $119-6=113$ dimensional surface in the 119-dimensional space $\mathscr{P}^{5}$. In contrast, $P_{\text {Borda }}$ (Definition 10) eliminates all dimensional restrictions on $\mathscr{C} \mathscr{B}^{3}$.

By being a smooth lower dimensional submanifold (i.e., a smooth surface) of profile space, $\mathscr{C} \mathscr{B}^{3}$ constitutes an unlikely event (i.e., a set with Lebesgue measure zero). Consequently, it is unlikely for an arbitrarily selected profile to satisfy even the binary part of the choice axiom. As "unlikely assertions" are standard for profile restrictions, different restrictions can be compared in terms of the dimensions of their spaces of admitted profiles. This dimensional comparison shows that $\mathscr{C} \mathscr{B}^{n}$ is a reasonably relaxed restriction. For instance, as Black's (1958) condition (imposed in voting to achieve binary consistency) for three alternatives excludes the two voter types (hence two dimensions) where a particular alternative is bottom-ranked, it defines a three-dimensional surface of profiles. With the three choices of the specified alternative, Black's condition defines the union of three three-dimensional submanifolds. By excluding two dimensions, rather than only one, Black's approach constitutes a more severe profile restriction than $\mathscr{C} \mathscr{B}^{3}$. But even though Black's condition is stricter, it does not achieve the numerical precision of Luce's system and it allows many ranking inconsistencies (Saari \& Valgones, 1999).

\subsection{Representation cube}

A factor that complicates determining the geometric structure of $\mathscr{C} \mathscr{B}^{n}$ is its large dimension. Therefore, rather than a direct analysis, I develop an indirect approach to exploit the linearity of pairwise tallies. The idea is that because the tally is linear, the pairwise outcomes inherit certain geometric traits of $\mathscr{C} \mathscr{B}^{n}$. For instance, if the pairwise outcomes (the image set) fail to have certain convexity properties, then $\mathscr{C} \mathscr{B}^{n}$ also fails to have these structures. In this indirect manner, we prove that $\mathscr{C} \mathscr{B}^{n}$ is a nonlinear submanifold (that is, a nonlinear surface).

While the approach and conclusions hold for any number of alternatives, I emphasize $n=3$ alternatives primarily because these conclusions can be described in terms of the familiar three-dimensional geometry. To do so, I use the representation cube (Saari, 1995) defined by the coordinates $\left(x_{A, B}, x_{B, C}, x_{C, A}\right)$ where

$x_{U, V}=P(U, V)-P(V, U), \quad U, V=A, B, C$.

It follows that

$-1 \leqslant x_{U, V} \leqslant 1, \quad x_{U, V}=-x_{V, U}$,

where $x_{U, V}=1,-1$ represent, respectively, perfect discrimination in selecting $U$ and $V$.
For any $\mathbf{p} \in \mathscr{P}^{3}$, the associated $\mathbf{x}=\left(x_{A, B}, x_{B, C}, x_{C, A}\right)$ resides in the orthogonal cube; this is the $R^{3}$ cube $[-1,1]^{3}$ defined by the eight vertices $( \pm 1, \pm 1, \pm 1)$. Let $\mathbf{E}_{j} \in \mathscr{P}^{3}$ be the probability defined by $p_{j}=1$; namely, $\mathbf{E}_{j}$ is where a selection is made from each pair with perfect discrimination. Thus the six probabilities $\left\{\mathbf{E}_{j}\right\}_{j=1}^{6}$ define the six vertices

$\begin{array}{cccc}\text { Probability } & \text { Vertex } V_{j} & \text { Probability } & \text { Vertex } V_{j} \\ \mathbf{E}_{1} & (1,1,-1) & \mathbf{E}_{4} & (-1,-1,1) \\ \mathbf{E}_{2} & (1,-1,-1) & \mathbf{E}_{5} & (-1,1,1) \\ \mathbf{E}_{3} & (1,-1,1) & \mathbf{E}_{6} & (-1,1,-1)\end{array}$

As $\mathbf{p}=\left(p_{1}, \ldots, p_{6}\right) \in \mathscr{P}^{3}$ is the convex sum $\sum_{j=1}^{6} p_{j} \mathbf{E}_{j}$, the linearity of how $x_{U, V}$ is computed implies that each $\mathbf{x}$ is a linear combination of the vertices $\left\{\mathbf{V}_{j}\right\}_{j=1}^{6}$.

Definition 13. The representation cube for three alternatives, denoted by $\mathscr{R} \mathscr{C}^{3}$, is the convex hull defined by the vertices $\left\{\mathbf{V}_{j}\right\}_{j=1}^{6}$.

There is a representation cube for all $n \geqslant 4$, but because $\mathscr{R} \mathscr{C}^{n}$ is in a $\left(\begin{array}{l}n \\ 2\end{array}\right)$ dimensional space (sixdimensional for $n=4)$, indirect arguments are required to analyze its properties. (Readers interested in $\mathscr{R} \mathscr{C}^{n}$ should consult Saari (2000a).) The representation cube $\mathscr{R} \mathscr{C}^{3}$, depicted in Fig. 8, starts with an orthogonal cube. Only six of the eight vertices represent transitive unanimity profiles, so two of them never arise with probability comparisons. One, $(1,1,1)$, represents the impossible cyclic outcome where each of $A \succ B, B \succ$ $C, C \succ A$ occurs with perfect discrimination. (The other vertex, $(-1,-1,-1)$ represents the reversed cycle.) To convert the orthogonal cube into $\mathscr{R} \mathscr{C}^{3}$, connect the relevant unanimity vertices with lines and flat surfaces; this construction excludes the two small tetrahedrons that have the cyclic vertices.

Proposition 5. With a specified linear computational rule for pairs, each $\mathbf{p} \in \mathscr{P}^{n}$ defines a unique point $\mathbf{x} \in \mathscr{R}^{n}$. Conversely, for each $\mathbf{x} \in \mathscr{R} \mathscr{C}^{n}$ and with the standard

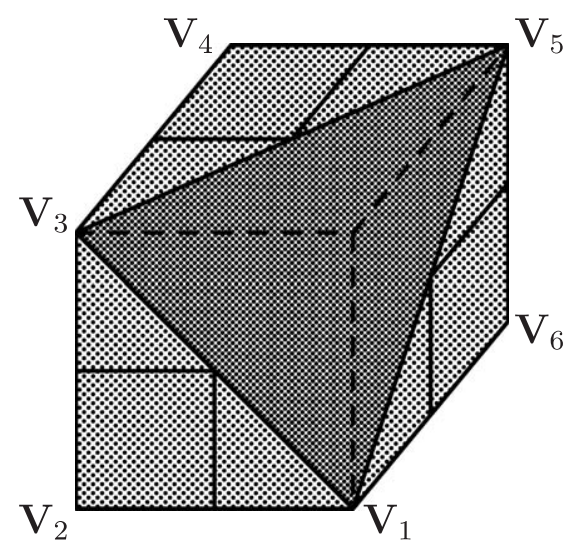

Fig. 8. Representation Cube. 
computational approach, there exist $\mathbf{p} \in \mathscr{P}^{n}$ that define $\mathbf{x}$. If no coordinate of $\mathbf{x} \in \mathscr{R} \mathscr{C}^{n}$ is \pm 1 (i.e., no perfect discrimination), then with the standard approach, $\mathbf{x}$ is supported by a $\left(n !-1-\left(\begin{array}{l}n \\ 2\end{array}\right)\right)$-dimensional linear subspace of $\mathscr{P}^{n}$.

For $n=3$, Proposition 5 asserts that if $\mathbf{x} \in \mathscr{R} \mathscr{C}^{3}$ is not on a face of the orthogonal cube (which implies perfect discrimination for some pair), then $\mathbf{x}$ is supported by a two-dimensional continuum of profiles. To illustrate, because $\mathbf{p}_{1}=\left(\frac{1}{4}, \frac{1}{5}, 0, \frac{1}{5}, \frac{7}{20}, 0\right), \mathbf{p}_{2}=\left(\frac{1}{5}, \frac{3}{20}, \frac{1}{10}, \frac{3}{20}, \frac{3}{10}, \frac{1}{10}\right)$, and $\mathbf{p}_{3}=\left(\frac{1}{4}, \frac{1}{20}, \frac{3}{20}, \frac{1}{5}, \frac{1}{5}, \frac{3}{20}\right)$ are linearly independent and each (with the standard pairwise computation) defines $\mathbf{x}=$ $\left(-\frac{1}{10}, \frac{1}{5}, \frac{1}{10}\right)$, the two-dimensional subspace of profiles

$\left\{\mathbf{p} \in \mathscr{P}^{3} \mid \mathbf{p}=(1-s-t) \mathbf{p}_{1}+s \mathbf{p}_{2}+t \mathbf{p}_{3}\right.$,

for all $s, t$ where $\left.\mathbf{p} \in \mathscr{P}^{3}\right\}$

also defines $\mathbf{x}$. This $n=3$ alternative, two-dimensional space is spanned by the reversal vectors defined in Section 2.

Recall that $\mathbf{R}_{X}$ (Definition 6) adds zero points to any candidate's pairwise tally, so the reversal terms do not affect pairwise tallies. But the sum of the $\mathbf{R}_{X}$ components is zero, so $\mathbf{p}^{*}=\mathbf{p}+\gamma \mathbf{R}_{X}$ defines another probability profile as long as $\gamma$ is selected so that all $\mathbf{p}^{*}$ components are non-negative. As already described (Section 2), these $\mathbf{R}_{X}$ vectors span a two-dimensional space and form the kernel of the standard pairwise computations for $n=3$ alternatives.

Corollary 3. For $n=3$, let $\mathbf{p}$ be a specified profile and let $\gamma_{A}, \gamma_{B}, \gamma_{C}$ be scalars so that

$\mathbf{p}^{*}=\mathbf{p}+\sum_{X=A, B, C} \gamma_{X} \mathbf{R}_{X}$

also is in $\mathscr{C} \mathscr{B}^{3}$. For the standard computation of pairwise votes $\mathbf{p}$ and $\mathbf{p}^{*}$ have the same pairwise tallies for all pairs. Conversely, if $\mathbf{p}^{*}$ and $\mathbf{p}$ have the same pairwise tallies, then they are related in Eq. (7.3) form. Thus, $\mathbf{p} \in \mathscr{C} \mathscr{B}^{3}$ if and only if $\mathbf{p}^{*} \in \mathscr{C} \mathscr{B}^{3}$.

This pairwise voting kernel is two-dimensional for three alternatives, and its dimension rapidly increases with the number of alternatives; e.g., each $\mathscr{R} \mathscr{C}^{5}$ outcome (that is, specific probabilities for each of the ten pairs coming from five alternatives) is supported by not a single profile, but by a 109-dimensional space of profiles. Imagine the enormous potential flexibility for modeling that is admitted by this 109-dimensional jump.

By combining Proposition 5 with Theorem 12, we see that $\mathscr{C} \mathscr{B}^{n}$ can be thought of as the product of an $(n-1)$ dimensional manifold with the $\left(n !-1-\left(\begin{array}{l}n \\ 2\end{array}\right)\right)$-dimensional pairwise voting kernel. To understand the geometric structure of $\mathscr{C} \mathscr{B}^{n}$, it suffices to identify the pairwise voting kernel (Corollary 3) and a particular $(n-1)$-dimensional portion of $\mathscr{C} \mathscr{B}^{n}$.
To demonstrate that $\mathscr{C} \mathscr{B}^{3}$ is very nonlinear, note from Definition 12 and Eq. (7.1) that the binary outcomes in $\mathscr{R} \mathscr{C}^{3}$ allowed by $\mathscr{C} \mathscr{B}^{3}$ profiles define the surface

$\mathbf{x}=\left(\frac{a-b}{a+b}, \frac{b-c}{b+c}, \frac{c-a}{c+a}\right) \in \mathscr{R} \mathscr{C}^{3}$.

By using $a+b+c=1$, the parametric representation of the two-dimensional surface of pairwise outcomes in $\mathscr{R} \mathscr{C}^{3}$ generated by $\mathscr{C} \mathscr{B}^{3}$ is

$\mathbf{x}=\left(\frac{a-b}{a+b}, \frac{2 b+a-1}{1-a}, \frac{1-2 a-b}{1-b}\right)$,

$$
a \geqslant 0, b \geqslant 0, a+b \leqslant 1 \text {. }
$$

Theorem 13. The pairwise outcomes of $\mathscr{C} \mathscr{B}^{3}$ define a smooth, two-dimensional non-linear surface in $\mathscr{R} \mathscr{C}^{3}$. Using $(x, y, z)$ as the $\mathscr{R} \mathscr{C}^{3}$ coordinates, the hyperbolic surface is $x y z+x+y+z=0$.

Proof. As Eq. (7.4) is a parametric representation of the $\mathscr{C} \mathscr{B}^{3}$ pairwise outcomes, Eq. (7.5) follows from elementary algebra.

A convenient way to visualize the geometry of the $\mathscr{C} \mathscr{B}^{3}$ pairwise outcomes is to hold one Eq. (7.5) variable fixed and then describe the resulting curve - an hyperbola. As Eq. (7.5) is symmetric in the three variables, the analysis is the same for whichever variable is held fixed. Fig. 9 displays three sections of this surface.

The symmetry of the $\mathscr{C} \mathscr{B}^{3}$ pairwise outcomes is displayed in Fig. 9. For instance, when $x=0$ (meaning a $A \sim B$ pairwise tie, or indifference), the section is the straight line $y+z=0$. Choosing $x$ values that differ only by sign, as in Figs. $9 \mathrm{~b}$ and $\mathrm{c}$, the resulting sections of $\mathscr{C} \mathscr{B}^{3}$ pairwise outcomes differ by a reflection about the $y+z=0$ diagonal line. (Similar symmetries hold for any number of alternatives.)

When $x \rightarrow 1$ (that is, when approaching perfect discrimination of $A$ over $B$ ), the curved hyperbola bends to become the union of two particular edges of the $x=1$ face of $\mathscr{R} \mathscr{C}^{3}$. Similarly, as $x \rightarrow-1$ the limit is two of the edges of the $x=-1$ face of $\mathscr{R} \mathscr{C}^{3}$. (These are the two edges that can be drawn without using the cyclic vertex.)

Corollary 4. The intersection of the closure of the surface of $\mathscr{C} \mathscr{B}^{3}$ pairwise outcomes with the $\mathscr{R} \mathscr{C}^{3}$ surface is the

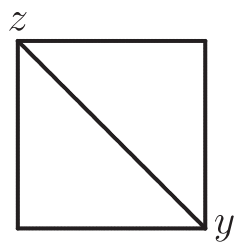

(a) $x=0$

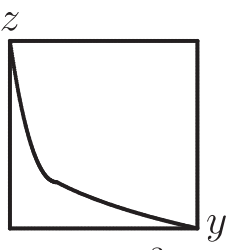

(b) $\quad x=\frac{3}{4}$

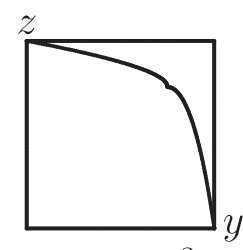

(c) $x=-\frac{3}{4}$
Fig. 9. Sections of the $\mathscr{L}^{3}$ pairwise outcome space. 
union of the six $\mathscr{R} \mathscr{C}^{3}$ edges connecting vertex $\mathbf{V}_{1}$ to $\mathbf{V}_{2}$ to ... to $\mathbf{V}_{6}$ to $\mathbf{V}_{1}$.

(For $n>3$ alternatives, the edges are replaced with higher-dimensional $\mathscr{R} \mathscr{C}^{n}$ surfaces.)

Proof. On the $x=1$ face, Eq. (7.5) becomes $y z+y+$ $z+1=(y+1)(z+1)=0$. This equation is satisfied if $y, z=-1$. This defines the equations for two of the edges.

According to Corollary 4, (3) of the choice axiom is a boundary condition introduced to ensure continuity for (2) of the axiom. Namely with perfect $P(A, B)=1$ discrimination given by $x=1$, rather than allowing any outcome from the full $x=1$ face, only those from two edges are allowed. Using Fig. 8 to envision the $\mathscr{C} \mathscr{B}^{3}$ pairwise outcomes, the surface starts as the union of two edges from the $x=1 \mathscr{R} \mathscr{C}^{3}$ face. As $x$ decreases in value, the corresponding section is a hyperbola with ends on the $\mathbf{V}_{3}-\mathbf{V}_{4}$ edge and $\mathbf{V}_{1}-\mathbf{V}_{6}$ edges of $\mathscr{R} \mathscr{C}^{3}$. When $x=0$, the section is a straight line. The surface for negative $x$ values is a reflection of what occurred for $|x|$ until it ends at $x=-1$, which consists of the two edges on the $x=$ -1 face (representing $P(B, A)=1$ ).

\subsection{Consequences}

If a subject doubts her original evaluation $\mathbf{p}_{1}$ of the alternatives because evidence suggests that $\mathbf{p}_{2}$ is a more accurate assessment, we might expect her to settle on some average. Stated in mathematical terms, it is reasonable to expect the four dimensions of $\mathscr{C}_{\mathscr{B}}{ }^{3}$ to admit an appropriate straight line of profiles. But this is not the case; instead, $\mathscr{C} \mathscr{B}^{3}$ is so nonlinear that it admits statements of the following kind. (This assertion extends to any number of alternatives.)

Corollary 5. With any linear computation of pairwise probabilities, the set $\mathscr{C} \mathscr{B}^{3}$ is not convex. Indeed, for any two profiles $\mathbf{p}_{1} \neq \mathbf{p}_{2}$ that satisfy the choice axiom with different pairwise probabilities and without ties, then, with the possible exception of one $\lambda \in(0,1)$ value, the profile

$\mathbf{p}_{3}=\lambda \mathbf{p}_{1}+(1-\lambda) \mathbf{p}_{2}$

does not satisfy the choice axiom.

Proof. Because $\mathbf{p}_{1}$ and $\mathbf{p}_{2}$ define different points on the $\mathscr{C} \mathscr{B}^{3}$ surface in $\mathscr{R} \mathscr{C}^{3}$ without ties, the outcomes are not on a linear portion of the surface. But a line connecting any two distinct points of this type on the surface can meet the surface in at most one other point. As the pairwise computations are linear, the pairwise outcome for $\mathbf{p}_{3}$ is in the interior of this line segment. This completes the proof.

According to Corollary 5, if a subject adheres to the choice axiom, she must exhibit curvilinear, rather than rectilinear, changes in profiles, ${ }^{4}$ changes that seem to be highly incompatible with the linear computations of probabilities. The curvature, and this curvilinear comment, are direct consequences of using the standard way to compute pairwise probabilities. In contrast, the $P_{\text {Borda }}$ (Definition 10) approach does allow rectilinear choices; by not suffering this limitation, we have another argument for using $P_{\text {Borda }}$.

This curvature can be understood by using Fig. 8 representation cube. In this cube, the basic profile outcomes (see Section 2) lie in what is called the transitivity plane (Saari, 1999) given by $x+y+z=0$. It turns out (Saari, 1999) that if a profile's outcome is not in the transitivity plane, then the profile must have a Condorcet component. As such, Fig. 9 proves that almost all $\mathscr{C} \mathscr{B}^{3}$ outcomes are out of the transitivity plane; e.g., the general case is for a $\mathscr{C} \mathscr{B}^{3}$ profile to have Condorcet components.

Now that the binary requirements of the choice axiom have been discussed, the next step is to identify the restrictions that are imposed on profiles with three alternative subsets. We already have done so in Section 3, but I now provide a more general approach. (For $n=3$, this completes the description of the profiles because the probability choices of each alternative being top-ranked defines the $a, b, c$ values.)

\subsection{Other probability decision rules}

As already noted, Luce's claim that his definition of $P_{T}(X)$ is logically independent of the choice axiom suggests that there must exist a wide selection of approaches. Earlier (Section 3) I introduced a continuum of choices different from that described by Luce: it remains to find a general result.

To do so in mathematical terms, express Eq. (1.8) as a smooth mapping

$F:\{(a, b) \mid 0 \leqslant a, b, a+b \leqslant 1\} \rightarrow \mathscr{P}^{3}$.

For Eq. (1.8), $F(a, b)=\mathbf{p}_{L}(a, b)$ is a point in the twodimensional set of profiles where the $\{A, B\}$ and $\{A, C\}$ respective pairwise probability values are $\left(\frac{a}{a+b}, \frac{b}{a+b}\right)$, $\left(\frac{a}{a+c}, \frac{c}{a+c}\right)$. As shown next, most mappings that can be expressed in an Eq. (7.6) format define a way to compute $P_{T}(X)$ that is compatible with the choice axiom.

Theorem 14. For $T=\{A, B, C\}$, almost all choices of smooth mappings Eq. (7.6), where $F(a, b)=\mathbf{p}$ is a probability profile with $\{A, B\},\{A, C\}$, and $\{B, C\}$ respective values of $\left(\frac{a}{a+b}, \frac{b}{a+b}\right),\left(\frac{a}{a+c}, \frac{c}{a+c}\right)$, and $\left(\frac{b}{b+c}, \frac{c}{b+c}\right)$ where

\footnotetext{
${ }^{4}$ As Luce reminded me, the (component wise) geometric mean $p_{1}^{\lambda} p_{2}^{1-\lambda}$ does satisfy the axiom. This product nature nicely captures the above curvilinear assertion about the nature of profiles that we now know is required by the choice axiom. Furthermore, it underscores a difference with other ways to compute probabilities that are discussed in Section 4.
} 
$c=1-a-b$, admit at least a local way to compute the probabilities $P_{T}\left(A_{j}\right)=a_{j}$ in a manner that satisfies the choice axiom.

Proof. Rather than using the implicit function theorem, the proof uses an explicit representation. To ensure that the pairwise computations hold, combine Corollary 3 with $\mathbf{p}_{L}(a, b)$ to express $F(a, b)=\left(p_{1}, \ldots, p_{6}\right)$ as

$F(a, b)=\mathbf{p}_{L}(a, b)+\alpha(a, b) \mathbf{R}_{A}+\beta(a, b) \mathbf{R}_{B}$.

To compute $P_{T}(A)$, add the ranking probabilities where $A$ is top ranked and a weighted " $s$ " multiple of the ranking probabilities where $A$ is second ranked. (In Fig. 6c, add an $s$ multiple of sum of the probabilities from the two heavier shaded regions to the sum of the probabilities from the lightly shaded regions.) The computational scheme for $P_{T}(A)$ requires choosing the value of $s$ so that

$a=a+\left\{2 \alpha-\beta+s\left[-2(2 \alpha-\beta)+\frac{c a}{1-c}+\frac{b a}{1-b}\right]\right\}$.

As long as the term in the square bracket is not zero, and this is true (at least locally) for almost all choices of $F$, an $s$ value can be found with the desired properties.

These alternative ways to compute $P_{T}(X)$ use information beyond those rankings where $X$ is topranked to include information (through the " $s$ " value) about when $X$ is second ranked. While Theorem 14 proves there are an uncountable number of ways to assign ranking probabilities with an associated $P_{T}(X)$ computation, it is unsatisfying from an esthetic and maybe a practical perspective. This is because the computational method, the value of $s$, may change with the $a$ and $b$ values and when computing $P_{T}(B)$ and $P_{T}(C)$. This blemish, of course, is avoided by using the Section 4 approach where a fixed $s$ weight is required for each $P_{T}(X)$ computation and all $a$ and $b$.

Theorem 14 holds for any linear pairwise computation method adopted to define $\mathscr{P}^{3}$. To illustrate by using the $\mathscr{P}^{3}$ space defined by the pairwise computational method of Eq. (1.14), instead of the standard Eq. (3.1), $F(a, b)$ assigns $p_{1}=\frac{3}{2}\left[\frac{a b}{a+b}-\frac{1}{18}\right]$ for the ranking $A \succ B \succ C$. To satisfy the choice axiom, the associated $P_{T}^{\prime}(A)$ is

$P_{T}^{\prime}(A)=\frac{7}{9}\left[p_{1}+p_{2}\right]+\frac{1}{9}\left[p_{3}+p_{4}+p_{5}+p_{6}\right]$.

More generally, $P_{T}^{\prime}(X), X=A, B, C$, is the $\frac{2}{3}$ multiple of the Luce-Plurality outcome of these particular ranking probabilities plus $\frac{1}{9}$. The point is not whether the resulting $P_{T}^{\prime}(X)$ is realistic, but rather to demonstrate that the choice axiom holds with a surprisingly rich class of ways to define probabilities.

\section{Concluding comment}

The natural sense captured by Luce's axiom, where a subject endows each alternatives with a certain level of intensity, probably explains a continued interest in the choice axiom nearly a half century after it was first introduced. But by adopting the perspective that the axiom implicitly defines all ways to compute probabilities along with the associated form of the ranking probabilities, it turns out that many structures and potential extensions of this axiom remain to be discovered. Indeed, while the geometric approach introduced here leads to a richer selection of alternative computational approaches, where the subject uses more information, and a significant relaxation on the choice of ranking probabilities, it is only an indication of what is possible. Also, by examining the information being used to make decisions, or compute probabilities, a different explanation of the reversal problem is obtained: it casts doubt on the standard way to compute $P(A, B)$ and other probabilities.

\section{Proofs}

Proof of Theorem 12. The proof uses the codimension argument of the standard implicit function theorem. The general setting, used in singularity theory (e.g., see Golubitsky \& Guillemin, 1973) has a smooth mapping $H: R^{m} \rightarrow R^{n}$ where for a smooth submanifold $\Sigma \subset R^{n}$ we wish to find the dimension of $H^{-1}(\Sigma)$. If $H$ has a transverse intersection with $\Sigma$ (that is, where the image of $H$ meets $\Sigma$, the tangent space for $\Sigma$ and $D H\left(R^{m}\right)$ span $R^{n}$ ), then the codimension of $H^{-1}(\Sigma)$ in $R^{m}$ equals the codimension of $\Sigma$ in $R^{n}$.

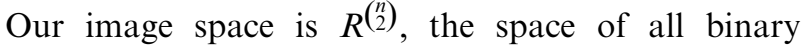
outcomes, while $H$ is the mapping computing these outcomes. We know from Saari (1995) that the image has an open set and that the transversality condition is satisfied. If $\Sigma$ is the set of choice binary outcomes, it is of dimension $n-1$ or codimension $\left(\begin{array}{l}n \\ 2\end{array}\right)-(n-1)$. The conclusion now follows.

Proof of Proposition 5. We need to prove that with the standard pairwise computation, each $\mathbf{x} \in \mathscr{R} \mathscr{C}^{n}$ is supported by a $\mathscr{P}^{n}$ subspace of codimension $\left(\begin{array}{l}n \\ 2\end{array}\right)$. The argument is the same as the above proof for Theorem 12 where $\Sigma$ now is the point $\mathbf{x}$ that has codimension $\left(\begin{array}{l}n \\ 2\end{array}\right)$. The transversality condition follows from the construction of $\mathscr{R} \mathscr{C}^{n}$.

Proof of Corollary 3. That the reversal terms are in the kernel follows from a direct computation. The proof that this is the total kernel follows from Saari (1999) and the profile decomposition described in Theorem 3. 
Proof of Theorem 4. As the assertion about the dimension of the kernel follows from Saari(2000a,b), it remains to show that Eq. (3.4) profiles satisfy Luce's conditions; i.e., that $P_{T}\left(A_{j}\right)=a_{j}$ and $P_{S}\left(A_{j}\right)=$ $\frac{P_{T}\left(A_{j}\right)}{\sum_{A_{k} \in S} P_{T}\left(A_{k}\right)}$. A proof of this assertion is in Luce \& Suppes (1965), but for completeness, a proof is given here. To prove that $P_{T}\left(A_{j}\right)=a_{j}$, it suffices to prove that $P_{T}\left(A_{1}\right)=a_{1}$, and it suffices to consider $A_{1} \succ A_{2} \succ \cdots \succ$ $A_{n}$ as a representative ranking. What simplifies the validation is that the form of $\mathscr{R}$ is

$$
\begin{aligned}
\mathscr{R} & \left(A_{1} \succ \cdots \succ A_{n}\right) \\
& =a_{1} \times \frac{a_{2}}{1-a_{1}} \times \cdots \times \frac{a_{n-1}}{1-\sum_{1}^{n-2} a_{j}} \\
& =P_{T}\left(A_{1}\right) P_{S_{n-1}}\left(A_{2}\right) \ldots P_{S_{n-(n-3)}}\left(A_{n-2}\right) P\left(A_{n-1}, A_{n}\right),
\end{aligned}
$$

where $S_{n-k}=\left\{A_{k+1}, \ldots, A_{n}\right\}$. Hence, properties of the probabilities simplify the computations. This is indicated with the computations carried out next.

To verify this assertion $P_{T}\left(A_{1}\right)=a_{1}$, I show that if $n-k$ of the $n$ alternatives are selected and ranked in the first $n-k$ position, say $A_{1} \succ A_{2} \succ \cdots \succ A_{n-k}$, then the sum of the ranking probabilities for the $k$ ! ways to complete the ranking with all alternatives, that is $\sum_{\sigma} \mathscr{R}\left(A_{1} \succ \cdots \succ A_{n-k} \succ \sigma\right)$ where $\sigma$ is a ranking of the alternatives $\left\{A_{n-k+1}, \ldots, A_{n}\right\}$, has the form

$$
\begin{aligned}
& \sum_{\sigma} \mathscr{R}\left(A_{1} \succ \cdots \succ A_{n-k} \succ \sigma\right) \\
& \quad=a_{1} \frac{a_{2}}{1-a_{1}} \cdots \frac{a_{n-k}}{1-\sum_{j=1}^{n-(k+1)} a_{j}} .
\end{aligned}
$$

Since Eq. (9.2) is of the $P_{T}\left(A_{1}\right) P_{S_{n-1}}\left(A_{2}\right) \cdots P_{S_{n-(n-(k+1))}}\left(A_{n-k}\right)$ form, to change Eq. (9.1) into Eq. (9.2) form, the approach is to sum over all alternatives in $S_{n-(n-(k+1))}=\left\{A_{n-k}, \ldots, A_{n}\right\}$ to cancel probability terms at the end of this expression. This is what is done.

For $k=2$, there are two ways to rank the last two alternatives $A_{n-1}$ and $A_{n}$. The sum of the two ranking probabilities has the common factor $a_{1} \frac{a_{2}}{1-a_{1}} \cdots \frac{a_{n-2}}{1-\sum_{j=1}^{n-3} a_{j}}$, defined by the common ranking of the first $n-2$ alternatives, times $\left(a_{n-1}+a_{n}\right) /\left(1-\sum_{j=1}^{n-2} a_{j}\right)$. As $\sum a_{j}=1$, this last term equals unity, so the sum of the ranking probabilities is the common factor that is the $k=2$ version of Eq. (9.2). In Eq. (9.1) terms, we have $\mathscr{R}\left(A_{1} \succ \cdots \succ A_{n-1} \succ A_{n}\right)+\mathscr{R}\left(A_{1} \succ \cdots \succ A_{n} \succ\right.$ $\left.A_{n-1}\right)=P_{T}\left(A_{1}\right) P_{S_{n-1}}\left(A_{2}\right) \cdots P_{S_{n-(n-3)}}\left(A_{n-2}\right)\left[P\left(A_{n-1}, A_{n}\right)+\right.$ $\left.P\left(A_{n}, A_{n-1}\right)\right]$, where the term in the brackets must equal unity. The same approach is used in an iterative fashion.

Assume Eq. (9.2) holds for $k=i$; we show that it holds for $k=i+1$. Since it holds for $k=i$, for each ranking of the first $n-(i+1)$ alternatives, there are precisely $i+1$ ways to add the next alternative $A_{\alpha}$. According to the induction hypothesis, the sum of the ranking probabilities for each way to complete the ranking is $\left[a_{1} \frac{a_{2}}{1-a_{1}} \cdots \frac{a_{n-(i+1)}}{1-\sum_{j=1}^{n-((i+1)+1)} a_{j}}\right] \frac{a_{\alpha}}{1-\sum_{j=1}^{n-(i+1)} a_{j}}$. The sum over all of these values has the common factor in the brackets times $\sum_{\alpha=n-i}^{n} a_{\alpha} /\left[1-\sum_{j=1}^{n-(i+1)} a_{j}\right]$. Again, since $\sum a_{j}=1$, the last term equals unity, so the final value is the common factor; this is Eq. (9.2) for $k=$ $i+1$, which completes the induction hypothesis. The $P\left(A_{1}\right)=a_{1}$ assertion follows from Eq. (9.2) for $k=$ $n-1$.

Again, using Eq. (9.1) formulation to explain this computation, Eq. (9.2) for $k=i$ becomes $P_{T}\left(A_{1}\right) P_{S_{n-1}}\left(A_{2}\right) \cdots P_{S_{n-(n-(i+2))}}\left(A_{n-(i+1)}\right) P_{S_{n-(n-(i+1))}}\left(A_{n-i}\right)$. The goal is to replace the last $A_{n-i}$ with all choices from $S_{n-(n-(i+1))}=\left\{A_{n-i}, \ldots, A_{n}\right\}$ and take the sum. This leads to $P_{T}\left(A_{1}\right) P_{S_{n-1}}\left(A_{2}\right) \cdots P_{S_{n-(n-(i+2))}}\left(A_{n-(i+1)}\right)$ times a sum of terms that must add to unity. To verify that $P_{S}\left(A_{j}\right)=\frac{P_{\left.T^{(} A_{j}\right)}}{\sum_{A_{k} \in S} P_{T}^{\left(A_{k}\right)}}$, it suffices to
prove that

$P_{S_{n-k}^{*}}\left(A_{1}\right)=\frac{a_{1}}{\sum_{j=1}^{n-k} a_{j}}, \quad S_{n-k}^{*}=\left\{A_{1}, \ldots, A_{n-k}\right\}$.

To explain the difference between Eqs. (9.2) and (9.3) by using Fig. 1a, notice that $P_{T}(A)$ is the sum of the ranking probabilities of regions 1 and 2 while $P(A, B)$ is the sum of the ranking probabilities of regions 1,2 , and 3 ; hence, with Eq. (9.1),

$P(A, B)=P_{T}(A)+P_{T}(C) P(A, B)$.

What is needed is to establish that this equation holds.

For $k=1, P_{S_{n-1}^{*}}\left(A_{1}\right)$ is the sum of all ranking probabilities where $A_{1}$ is top ranked plus those where $A_{1}$ is second ranked and $A_{n}$ is top ranked; notice, this last sum is $P_{T}\left(A_{n}\right) P_{S_{n-1}^{*}}\left(A_{1}\right)$ for whatever form $P_{S_{n-1}^{*}}\left(A_{1}\right)$ assumes. As established above, the first sum is $a_{1}$. The sum of ranking probabilities where $A_{1}$ is second ranked and $A_{n}$ is top ranked is, according to Eq. (9.2), $a_{n} \frac{a_{1}}{1-a_{n}}$. Thus $P_{S_{n-1}^{*}}\left(A_{1}\right)=a_{1}+a_{n} \frac{a_{1}}{1-a_{n}}=\frac{a_{1}}{1-a_{n}}$, which is Eq. (9.3) for $k=1$. Restating this computation in Eq. (9.4) terms, we must establish that the specified form of $P_{S_{n-1}^{*}}\left(A_{1}\right)$ equals $P_{T}\left(A_{1}\right)+P_{T}\left(A_{n}\right) P_{S_{n-1}^{*}}\left(A_{1}\right)$, or that $P_{T}\left(A_{1}\right) /[1-$ $\left.P_{T}\left(A_{n}\right)\right]$ has the predicted $P_{S_{n-1}^{*}}\left(A_{1}\right)$ form; a quick calculation proves that it does.

Assuming that Eq. (9.3) holds for $k=i$, we show that it holds for $k=i+1$. Here, $P_{S_{n-(i+1)}^{*}}\left(A_{1}\right)=P_{S_{n-i}^{*}}\left(A_{1}\right)+$ $K P_{S_{n-i}^{*}}\left(A_{n-i}\right)$ where $K P_{S_{n-i}^{*}}\left(A_{n-i}\right)^{n-(i+}$ denotes the partial computation of $P_{S_{n-i}^{*}}\left(A_{n-i}\right)$, which excludes those ranking probabilities where alternatives from $S_{n-(i+1)}^{*}$ are ranked above $A_{1}$. By use of Eq. (9.1), whatever the form of $P_{S_{n-(i+1)}^{*}}\left(A_{1}\right)$, we have that $K P_{S_{n-i}^{*}}\left(A_{n-i}\right)=$ 
$P_{S_{n-i}^{*}}\left(A_{n-1}\right) P_{S_{n-(i+1)}^{*}}\left(A_{1}\right)$. Thus, we need to determine whether $P_{S_{n-i}^{*}}\left(A_{1}\right) /\left[1-P_{S_{n-i}^{*}}\left(A_{n-1}\right)\right]$ has the predicted representation for $P_{S_{n-(i+1)}^{*}}\left(A_{1}\right)$. According to the induction hypothesis

$\frac{P_{S_{n-i}^{*}}\left(A_{1}\right)}{1-P_{S_{n-i}^{*}}\left(A_{n-1}\right)}=\frac{a_{1} / \sum_{j=1}^{n-i} a_{j},}{1-\left[a_{n-i} / \sum_{j=1}^{n-i} a_{j}\right]}=\frac{a_{1}}{a_{1}+\cdots+a_{n-(i+1)}}$

is the desired expression.

Proof of Corollary 1. Because Eq. (4.4) can be expressed in an Eq. (9.1) format, the proof is essentially the same as the proof of Theorem 4 given above.

\section{Acknowledgments}

This research was supported by NSF Grant DMI 0233798. My thanks to D. Luce for introducing me to this topic and for his several useful suggestions and comments about the earlier drafts of this paper. Also, my thanks L. Narens for conversations and correspondence about this subject, and to an anonymous referee who made several useful suggestions including that the paper be reorganized by starting and placing more emphasis on recent results in "voting theory."

\section{References}

Arrow, K. J. (1963). Social choice and individual values (2nd ed.). New York: Wiley.

Black, D. (1958). The theory of committees and elections. London: Cambridge University Press.

Block, H. D., \& Marschak, J. (1960). Random orderings and stochastic theories of responses. In I. Olkin, S. Ghurye, W. Hoeffding, W. Madow, \& H. Mann (Eds.), Contributions to probability and statistics (pp. 97-132). Stanford: Stanford University Press.

Debreu, G. (1960). Review of 'Individual choice behavior: A theoretical analysis'. American Economic Review, 50, 186-188.

Estes, W. K. (1997). Some reflections on the role of the choice model in theories of categorization, identification, and learning. In A. A. J. Marley, Choice, decision, and measurement: Essays in honor of $R$. Duncan Luce (pp. 321-346). New Jersey, NJ: Erlbaum Assoc.

Fishburn, P. (1994). On choice probabilities derived from ranking probabilities. Journal of Mathematical Psychology, 29, 274-285.

Fishburn, P. (2002). Stochastic utility. In S. Barbera, J. Hammon, \& C. Seidl (Eds.), Handbook of utility (pp. 275-319). Dordrecht/Boston: Kluwer Academic Publishers.

Golubitsky, M., \& Guillemin, V. (1973). Stable mappings and their singularities. Berlin: Springer.
Luce, R. D. (1959). Individual choice behavior. New York: Wiley.

Luce, R. D. (1961). A choice theory analysis of similarity judgments. Psychometrika, 26, 151-163.

Luce, R. D. (1962). An observable property equivalent to a choice model for discrimination experiments. Psychometrika, 27, 163-167.

Luce, R. D. (1977). The choice axiom after twenty years. Journal of Mathematical Psychology, 15, 215-233.

Luce, R. D., Bush, R. R., \& Galanter, E. (1965). Handbook of mathematical psychology (Vol. III). New York: Wiley.

Luce, R. D., \& Suppes, P. (1965). Preference, utility and subjective probability. In R. D. Luce, R. R. Bush, \& E. Galanter (Eds.), Handbook of mathematical psychology (Vol. 3, pp. 249-410). New York, NY: Wiley.

Marley, A. A. J. (1968). Some probabilistic models of simple choice and ranking. Journal of Mathematical Psychology, 5, 311-332.

Marley, A. A. J. (1982). Random utility models with all choice probabilities expressible as "functions" of the binary choice probabilities. Mathematical Social Science, 3, 39-56.

Marley, A. A. J. (1997). Choice, decision, and measurement: Essays in honor of R. Duncan Luce. New Jersey, NJ: Erlbaum Assoc.

Saari, D. G. (1989). A dictionary for voting paradoxes. Journal of Economic Theory, 48, 443-475.

Saari, D. G. (1995). Basic geometry of voting. New York: Springer.

Saari, D. G. (1992). Millions of election rankings from a single profile. Social Choice \& Welfare, 9, 277-306.

Saari, D. G. (1998). Connecting and resolving Arrow's and Sen's theorems. Social Choice \& Welfare, 15, 239-261.

Saari, D. G. (1999). Explaining all three alternative voting outcomes. Journal of Economic Theory, 87, 313-355.

Saari, D. G. (2000a). Explaining positional voting paradoxes I: The binary case. Economic Theory, 15, 1-53.

Saari, D. G. (2000b). Explaining positional voting paradoxes II: The general case. Economic Theory, 15, 54-103.

Saari, D. G. (2001a). Chaotic elections! A mathematician looks at voting. Providence, RI: American Mathematical Society.

Saari, D. G. (2001b). Decisions and elections: Explaining the unexpected. New York: Cambridge University Press.

Saari, D. G. (2002). Adopting a plurality vote perspective. Mathematics of Operations Research, 27, 45-64.

Saari, D. G., \& Sieberg, K. (2001). The sum of the parts can violate the whole. American Political Science Review, 95, 415-433.

Saari, D. G., \& Valgones, F. (1999). The geometry of Black's single peakedness condition. Journal of Mathematical Economics, 32, 429-456.

Tversky, A. (1972a). Elimination by aspects: A theory of choice. Psychological Review, 79, 281-299.

Tversky, A. (1972b). Choice by elimination. Journal of Mathematical Psychology, 9, 341-367.

Yellott, J. I., Jr. (1997a). The relationship between Luce's Choice Axiom, Thurston's theory of comparative judgement, and the double exponential distribution. Journal of Mathematical Psychology, 15, 109-144.

Yellott, J. I., Jr. (1997b). Preference models and irreversibility. In A. A. J. Marley (Ed.), Choice, decision, and measurement: Essays in honor of R. Duncan Luce (pp. 131-151). New Jersey, NJ: Erlbaum Assoc. 